\title{
Disease-linked microRNA-21 exhibits drastically reduced mRNA binding and silencing activity in healthy mouse liver
}

\author{
JOHN R. ANDROSAVICH, ${ }^{1,2}$ B. NELSON CHAU, ${ }^{3}$ BALKRISHEN BHAT, ${ }^{3}$ PETER S. LINSLEY, $, 3,4$ \\ and NILS G. WALTER ${ }^{1,5}$ \\ ${ }^{1}$ Department of Chemistry, ${ }^{2}$ Program in Chemical Biology, University of Michigan, Ann Arbor, Michigan 48109-1055, USA \\ ${ }^{3}$ Regulus Therapeutics, San Diego, California 92121, USA
}

\begin{abstract}
MicroRNAs (miRNAs) bind to mRNAs and fine-tune protein output by affecting mRNA stability and/or translation. miR-21 is a ubiquitous, highly abundant, and stress-responsive miRNA linked to several diseases, including cancer, fibrosis, and inflammation. Although the RNA silencing activity of miR-21 in diseased cells has been well documented, the roles of miR21 under healthy cellular conditions are not well understood. Here, we show that pharmacological inhibition or genetic deletion of miR-21 in healthy mouse liver has little impact on regulation of canonical seed-matched mRNAs and only a limited number of genes enriched in stress response pathways. These surprisingly weak and selective regulatory effects on known and predicted target mRNAs contrast with those of other abundant liver miRNAs such as miR-122 and let-7. Moreover, miR-21 shows greatly reduced binding to polysome-associated target mRNAs compared to miR-122 and let-7. Bioinformatic analysis suggests that reduced thermodynamic stability of seed pairing and target binding may contribute to this deficiency of miR-21. Significantly, these trends are reversed in human cervical carcinoma (HeLa) cells, where miRNAs including miR-21 show enhanced target binding within polysomes and where miR-21 triggers strong degradative activity toward target mRNAs. Taken together, our results suggest that, under normal cellular conditions in liver, miR-21 activity is maintained below a threshold required for binding and silencing most of its targets. Consequently, enhanced association with polysome-associated mRNA is likely to explain in part the gain of miR-21 function often found in diseased or stressed cells.
\end{abstract}

Keywords: microRNA-21; liver; HeLa cells; anti-miR inhibition of microRNA; array profiling; cancer

\section{INTRODUCTION}

MicroRNAs (miRNAs) are an expansive class of evolutionarily conserved post-transcriptional gene regulators effecting RNA silencing. At $\sim 21$ nucleotides (nt), these endogenous noncoding RNAs are partially complementary to the $3^{\prime}$ untranslated regions ( 3 ' UTRs) of mRNAs, and thus control and typically silence gene expression by recruiting proteins of the RNA induced silencing complex (RISC) to their mRNA targets (Lewis et al. 2003; Jackson and Standart 2007). A growing body of evidence has demonstrated that miRNAs are involved in nearly all processes of the cellular life cycle, from growth and differentiation to maintenance and cell death (Zhao and Srivastava 2007;

\footnotetext{
${ }^{4}$ Present address: AVI BioPharma, Bothell, WA 98021, USA

${ }^{5}$ Corresponding authors

E-mail nwalter@umich.edu

E-mail linsleyp@comcast.net

Article published online ahead of print. Article and publication date are at http://www.rnajournal.org/cgi/doi/10.1261/rna.033308.112.
}

Mallanna and Rizzino 2010; Vecchione and Croce 2010). This broad capacity is achieved by the actions of nearly 1500 miRNAs that are bioinformatically predicted to regulate expression of $>60 \%$ of the human proteome based on base-pairing rules involving 7- to 8-nt short "seed sequences" (Friedman et al. 2009). Perturbing the levels of a single miRNA has been shown to affect the mRNA and protein levels of hundreds of downstream genes (Baek et al. 2008; Selbach et al. 2008). Although the precise mechanism of miRNA-mediated regulation remains debated (Jackson and Standart 2007), global evaluation of expression changes following transfection with or deletion of miRNAs has revealed that, at least at later time points, the majority of changes observed at the protein level are mirrored at the transcript level, indicating that mRNA destabilization is an important component of miRNA function (Baek et al. 2008; Selbach et al. 2008; Hendrickson et al. 2009; Guo et al. 2010). Apparently, miRNA-mediated degradation can occur while the mRNA target is still actively translated within polyribosome (polysome) complexes (Kim et al. 2004; Nelson 
et al. 2004; Maroney et al. 2006; Nottrott et al. 2006; Jannot et al. 2011a).

Recently, the role of miRNAs in disease has become a strong focal point of research. To date, more than 4100 miRNA-disease associations have been reported ( $\mathrm{Lu}$ et al. 2008). While the interactions between a miRNA and its target mRNAs are believed to mostly result in mild "finetuning" of expression output, imbalances in this interplay can have deleterious effects on the cell. In some cases, dysregulation of a single miRNA-target interaction induces disease. For example, chromosomal truncations in the $3^{\prime}$ UTR of the mRNA encoding the human proto-oncogene high mobility group A2 (Hmga2), a transcriptional modulator expressed at high levels during embryogenesis but silenced in adulthood by let-7 miRNA, can lead to cancerous transformation due to loss of let-7 miRNA-mediated repression (Mayr et al. 2007). Alternatively, disease can arise from aberrant miRNA expression, which can potentially have much broader impacts on the transcriptome. For example, miR-21 is found to be overexpressed in several types of human tumors (Volinia et al. 2006) as well as heart fibroblasts following cardiac stress (Thum et al. 2008). While this correlation is suggestive of the pathological effects of miR-21, the miRNA's precise role in disease progression remains unresolved. Overexpression of miR-21 alone in the hematopoietic system can induce tumorigenesis in transgenic mice (Medina et al. 2010); however, in more solid tissues such as brain (Medina et al. 2010) and lung (Hatley et al. 2010), activation of another oncogene, such as K-ras (Hatley et al. 2010), appears necessary for miR-21 to contribute to and reinforce cancerous phenotypes.

Our knowledge of miRNA function and of the mechanistic links between miRNA and disease remains incomplete. In large part, this is due to poor understanding of the multitude of targets regulated by different miRNAs. While considerable effort has been expended toward global prediction of miRNA targets (Bartel 2009), experimental support for these predictions is limited. Indeed, most validated targets have been confirmed using gene reporter assays in cultured cancer cell lines. Little is known about miRNAs and their targets under healthy cellular conditions.

Here, we have investigated the function of three highly abundant miRNAs in healthy mouse liver. We show that, with the exception of a restricted set of genes enriched in stress-response pathways, inhibition of miR-21 with a specific "anti-miR" oligonucleotide drug or abrogation by genetic deletion has surprisingly little effect on mRNA levels of predicted and previously validated targets, in contrast to our observations for let-7 and miR-122. Consistent with a diminished repression capacity, miR-21 displays an unusual subcellular distribution with reduced binding to actively translating mRNAs. In stark contrast with healthy tissue, miR-21 displays a significantly augmented repression signature and strengthened binding to mRNA targets in the cancerous HeLa cell line, as previously suggested. Our analysis gives a biochemical perspective on the disparities of miRNA-mediated repression between healthy and diseased states and suggests that miR-2 1 passes a threshold in its transition from a very specific regulator under normal homeostasis to an overactive, broadly repressive oncomiR in cancer.

\section{RESULTS}

\section{miR-21 silences only a narrow subset of all mRNAs with matching seed sequence}

To study miR-21's intrinsic silencing activity in vivo in healthy animals, we used array profiling to measure changes in mouse liver mRNA populations resulting from inhibition of the miRNA. Anti-miR oligonucleotides were synthetically tailored to be complementary to the full-length miRNA and featured a number of chemical modifications to enhance specificity, affinity, and stability. We chose to focus on liver tissue, for the following reasons: (1) miR-21 is among the 10 most highly abundant miRNAs in both mouse and human liver samples (Supplemental Fig. S1; Edgar et al. 2002; Bissels et al. 2009; Burchard et al. 2010; Cheloufi et al. 2010); (2) anti-miR delivery to this tissue by systemic administration is made highly efficient by the hepatic portal system; and (3) miR-21 is found to be elevated in human hepatocellular carcinoma clinical samples and has been found to contribute to tumorigenesis and enhanced invasiveness by silencing the expression of the tumor suppressors Pdcd4, Pten, and Reck in hepatocyte-derived carcinoma cell lines (Liu et al. 2010). In addition to miR-21, we performed similar inhibition analyses on two other miRNAs that, similarly, are among the most highly expressed liver miRNAs (Supplemental Fig. S1): miR-122, formerly believed to be a liver specific miRNA, where it has been implicated in cancer and hepatitis C (Filipowicz and Grosshans 2011) but recently discovered to be expressed in other tissues as well (Burns et al. 2011); and let-7, a conserved family of 11 miRNA isoforms with high sequence similarity that is broadly involved in cell development and tumor suppression (Roush and Slack 2008).

All systemic anti-miR treatments had broad effects on the transcriptome with between $\sim 12 \%$ and $23 \%$ of the $>17,500$ genes measured being statistically significantly $(P<$ 0.05 by Student's $t$-test with three biological replicates per group) up- or down-regulated compared to a saline treatment as negative control (Fig. 1A, left). The majority of these changes were mild with median changes in the range of $14 \%-20 \%$ for down-regulated genes and $21 \%-31 \%$ for upregulated genes (Fig. 1A, right). Such changes may reflect shifts in the expression levels of miRNA targets, downstream effects caused by these shifts, and/or nonspecific effects of the anti-miR (Krützfeldt et al. 2005). To determine the primary effects of the anti-miR, that is, to elucidate the genes directly regulated by a miRNA, we binned genes based on the presence 
A

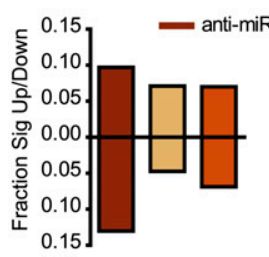

C
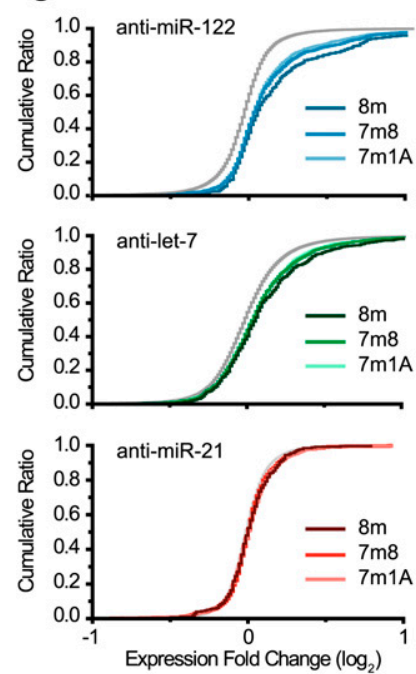

B

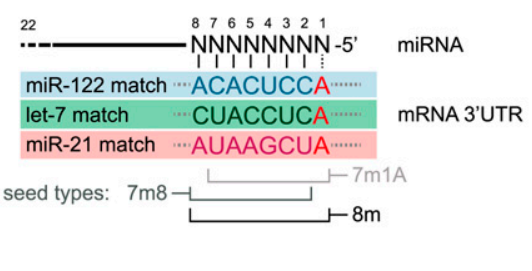

anti-let-7
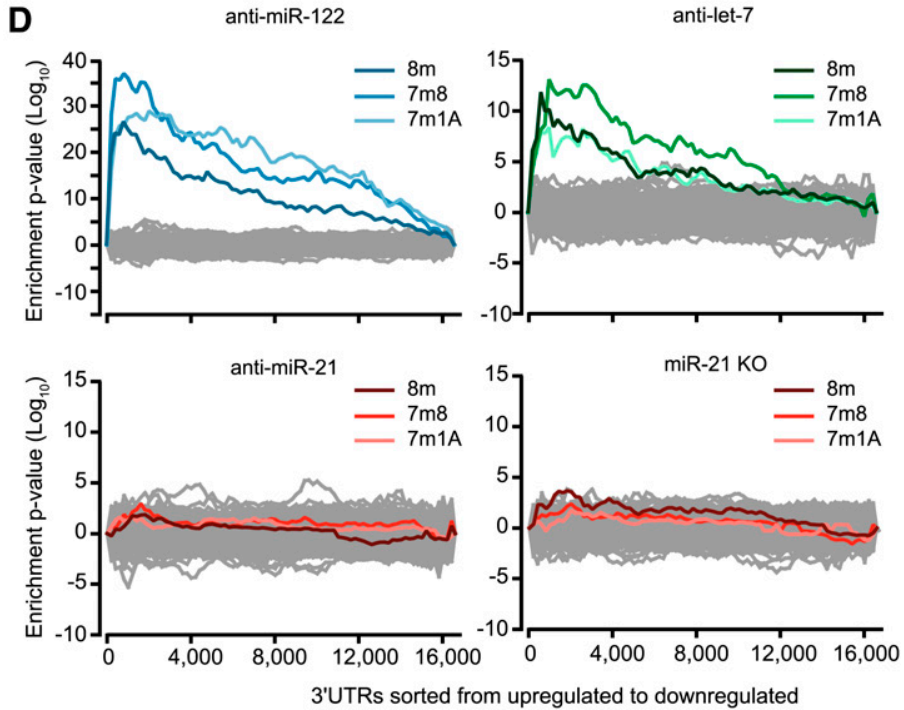

FIGURE 1. Array profiling of liver mRNA following miRNA inhibition or knockout. $(A)$ Total gene expression changes in response to anti-miR treatment. Left, fraction of total genes measured which were significantly $(P<0.05$, Student's $t$-test) up-regulated (positive $y$-axis) or downregulated (negative $y$-axis). Right, the magnitudes of change in expression for the same fractions plotted as cumulative distribution frequencies. The dotted line represents the median. (B) Schematic of canonical miRNA seed:mRNA seed-matched pairing for miR-122, let-7, and miR-21. The miRNA seed region, nucleotides $2-8$ in the $5^{\prime}$ end of a miRNA, binds to 7 - or 8-nt seed-matched sequences in the $3^{\prime}$ UTRs of mRNA transcripts. Three variations of seed matches have been identified as being the most critical for miRNA binding: heptanucleotide sequences complementary to either position $2-8$ of the miRNA $(7 \mathrm{~m} 8)$ or position $2-7$ with an adenosine at position $1(7 \mathrm{~m} 1 \mathrm{~A})$, or octanucleotide sequences complementary to position 2-8 plus an adenosine at position $1(8 \mathrm{~m})$. Note that while Watson-Crick base-pairing is not required across from position 1 , it frequently occurs since a majority of miRNAs contain uracils at this position. $(C)$ Cumulative distribution frequencies for all profiled genes plotted as a function of fold-change in expression following anti-miR treatment against miR-122 (top), let-7 (middle), or miR-21 (bottom). Genes were binned based on the presence (colored lines) or absence (gray line) of seed-matched sequences complementary to the inhibited miRNA. (D) Heptanucleotide Sylamer analysis of the same data sets shown in $C$ for each indicated treatment. The seed matches for each miRNA are highlighted. All other possible 7-nt sequences not related to the seed match are shown as gray lines and thus represent statistical background noise. The $8 \mathrm{~m}$ seed match from the octanucleotide analysis is shown superimposed. Heptanucleotide and octanucleotide analyses had similar backgrounds. The peaking of enrichment, calculated as a hypergeometric $P$-value, for seed-matched sequences on the left-hand side of the plot for miR-122 (top, left) and let-7 (top, right) indicates that the genes most up-regulated upon miRNA inhibition are enriched for the corresponding seed-matched sequence. Enrichment for miR-21 seed-matched genes is not observed when miR-21 is inhibited (bottom, left) or knocked out (bottom, right).

of 7 -nt (7m1A or $7 \mathrm{~m} 8)$ - or 8 -nt (8m)-long seed sequence matches in each mRNA's $3^{\prime}$ UTR that are complementary to the $5^{\prime}$ end of the miRNA (Fig. 1B). It is believed that $8 \mathrm{~m}-$ seed matches are the most effective sites, with mRNAs containing these sequences having the highest likelihood for being a miRNA target (Bartel 2009). In the case of miR122 and let-7, miRNA inhibition caused a significantly greater up-regulation of all seed-matched mRNA compared to nonmatched mRNA for the same cumulative fraction (one-sided Kolmogorov-Smirnov $P$-value $($ KS $P)=1.44 \times$ $10^{-25}$ for $8 \mathrm{~m}$-miR-122; $1.06 \times 10^{-8}$ for $8 \mathrm{~m}$-let-7) (Fig. 1C, top and middle panels). These results are consistent with both canonical miRNA-target predictions (Friedman et al. 2009) and previous results for miR-122 using similar experimental conditions (Elmén et al. 2008). Unexpectedly, this preferential effect on seed-matched targets was not strongly observed upon miR-21 inhibition (KS $P=3.74 \times 10^{-2}$ for $8 \mathrm{~m}$-miR21) (Fig. 1C, bottom). To verify this result, we reanalyzed the same profiling data using the Sylamer algorithm, which computes the hypergeometric significance for finding enrichment of short sequences on one end or the other of a profiling data set ranked from most up-regulated to most downregulated (van Dongen et al. 2008). The main advantage of the Sylamer algorithm is that it does not require a priori assumptions regarding sequence; it, instead, computes and plots enrichment signatures for all possible sequences of a certain input length, thus enabling nonspecific effects or other trends to be uncovered. Sylamer analysis showed that treat- 
ment with anti-miR-122 (Fig. 1D, top left, $P<1 \times 10^{-25}$ ) or let-7 (Fig. 1D, top right, $P<1 \times 10^{-12}$ ) induced upregulation of genes enriched for seed matches unique to the inhibited miRNA, thus demonstrating the specificity of these treatments. Still, we did not find enrichment on either end of the anti-miR-21 ranked gene list for miR-21 seed matches nor any other 7- or 8-nt sequence (Fig. 1D, bottom left), indicating that, on a transcriptomic scale, the primary effects of miR-21 inhibition cannot be clearly discerned above background.

The three anti-miR oligonucleotides used were identical in all chemical modifications but sequence, thus it is unlikely that the diminished effect of anti-miR-21 was due to drug inefficacy. To further rule out this possibility, we compared livers of wild-type and $m i R-21$ knockout mice (for validation, see $\mathrm{Lu}$ et al. 2011) and found a lack of derepression similar to inhibition by anti-miR-21 (Fig. 1D, bottom right).

We also noticed that, in contrast to the majority of targets containing 7- or 8-nt matches, only a narrow subset of miR-21 seed-matched transcripts were significantly upregulated upon miR-21 inhibition, restricted to just $248 \mathrm{~m}$ seed matches (Supplemental Table S1). This observation suggests that either miR-21 has a higher selectivity for these targets or it has fewer high-potential targets compared to miR-122 and let-7. We conclude that, in healthy liver tissue, miR-21 demonstrates very limited mRNA target engagement, silencing fewer targets to a lesser extent than other miRNAs of similarly high abundance.

\section{miR-21 repression corresponds poorly with targeting predictions and known targets}

We next sought to compare our experimental results with the most probable targets derived from the available target prediction databases. Not all mRNAs containing a seed match are expected to be repressed by a coexpressed miRNA, as the likelihood for an interaction can be dependent on several factors such as binding site accessibility and/or hybridization free energy. Several target prediction algorithms have been developed to take these factors into account, with the goal of decreasing the frequency of falsepositives (Krek et al. 2005; Griffiths-Jones et al. 2006; Grimson et al. 2007; Kertesz et al. 2007; Friedman et al. 2009; Betel et al. 2010; Garcia et al. 2011). Scores are assigned based on heavy weighting of local mRNA context features, evolutionary conservation, and/or thermodynamics in an effort to rank the most likely or most potent targets of a miRNA. Indeed, for miR-122 and let-7, we found that the experimental changes in mRNA level for the majority of top predicted targets across seven algorithms (see Materials and Methods) were, on average, greater than the changes for all 7- or 8-nt seed matches together (Fig. 2A, top), indicating that they were more predictive than just seed-matching alone. Our understanding of target prediction remains incomplete, as the mean changes of the top predicted targets were below those of the experimentally observed top-30 seed-matched targets, and several top predictions, particularly for let-7, were not significantly up-regulated upon anti-miR treatment (Fig. 2A, bottom). These observations, however, are in agreement with a similar comparison between top prediction sets and proteomic data following $m i R-223$ genetic deletion in mouse neutrophils (Baek et al. 2008), indicating a similar, if somewhat limited accuracy of the predicted responses for target proteins and mRNAs upon impairment of miR-223, miR-122, and let-7.

By comparison, we observed that far fewer predicted targets of miR-21 were regulated. Although a few targets were predicted correctly (Fig. 2A, bottom), these showed only very mild increases upon anti-miR treatment, while the majority of predicted targets were unresponsive or slightly downregulated (Fig. 2A, top). Surprisingly, none of the TargetScan predictions, among the most accurate for miR-122 and let-7, were correct for miR-21; the Context+ algorithm, which was designed to account for miRNAs with low 5' G/C content, a category into which miR-21 falls, did not significantly remedy this lack of accuracy (Fig. 2A).

A miRNA's base composition can have a significant influence on mRNA target binding capacity. Sequence analysis revealed full-length miR-21 as slightly more A/U-rich (64\%) than miR-122 (54\%) and let-7 (59\%). These differences are enhanced in the seed region: miR-21 (71\%), miR-122 (43\%), let-7d (43\%) (Fig. 1B). Since the seed region often accounts for the majority of miRNA:mRNA base-pairing interactions, we hypothesized that the release of free energy upon target binding would be much lower for miR-21 than for miR-122 or let-7. Indeed, computational analysis estimated the free energy of miR-21 binding to targets detected in our array profiling to be significantly less than for the other miRNAs (Fig. 2B), indicating that $3^{\prime}$ end pairing does not sufficiently compensate for weak seed pairing for a majority of targets. This reduced binding energy may partially explain miR-21's inactivity; however, we did not find much of a correlation between calculated binding energies and changes in mRNA levels (Supplemental Fig. S2A). Thermodynamics also does not satisfactorily explain why miR-21 does not repress Pdcd4, a validated target with extended complementary pairing and binding energies similar to those of miR-122 target AldoA (Supplemental Fig. S2B). Furthermore, the 5' miRNA seed region, critical for target recognition, may disproportionally contribute to the thermodynamics of target binding by serving to nucleate the miRNA:mRNA interaction. In line with a seednucleation binding model, structural probing of guide-strand loaded RISC suggests that the $5^{\prime}$ segment is preorganized by protein-RNA interactions in a conformation favorable for Watson-Crick base-pairing, while the $3^{\prime}$ end remains unconstrained, free to adopt a more flexible, less primed conformation (Lambert et al. 2011). Recent data from Bartel and colleagues emphasized the relationship between seed base 

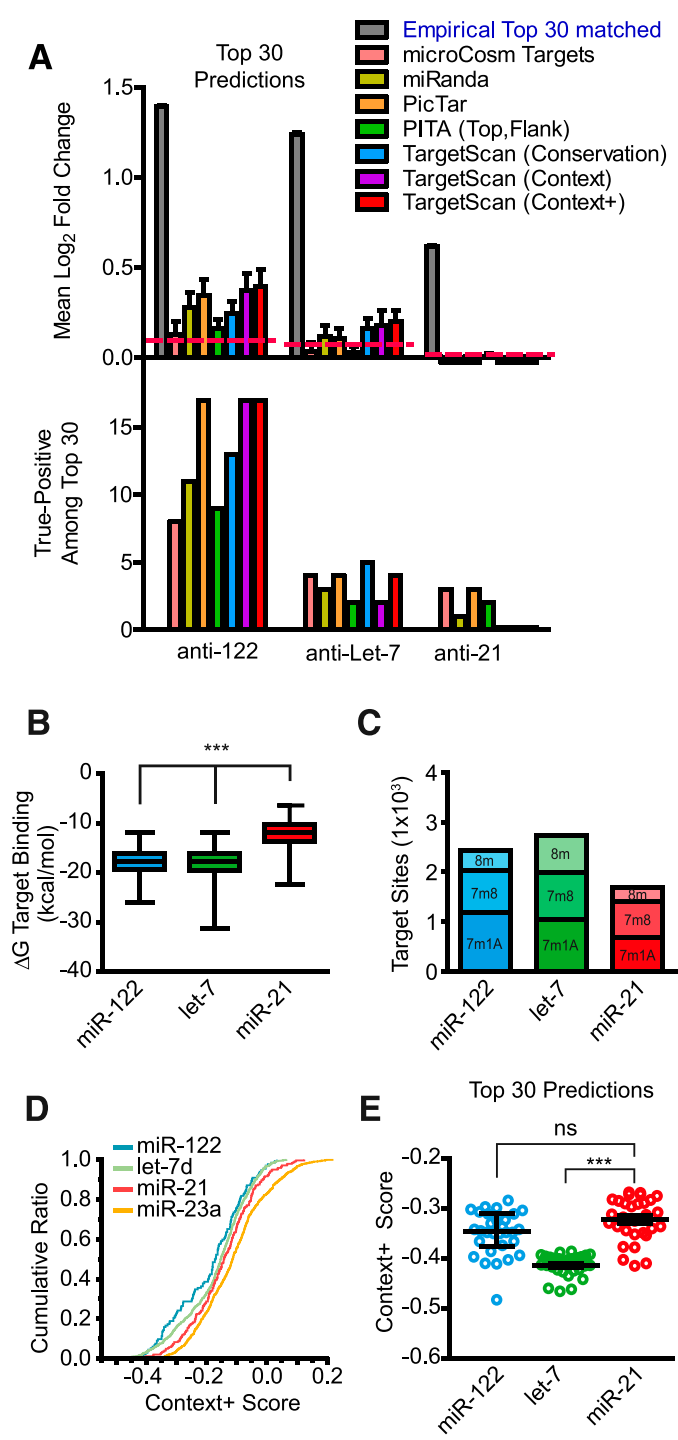

composition and repression by demonstrating that the low repression proficiencies of the Caenorhabditis elegans miRNA lsy- 6 and the human miRNA miR-23 were due, in part, to weak seed pairing; the other decisive factor was concluded to be high target abundance, which can dilute the effects a miRNA exerts on any given specific mRNA (Garcia et al. 2011). The influence of these factors was weighted in a modified version of the TargetScan context prediction scores, termed Context + . Interestingly, miR-21 and miR-23 seeds are similarly A+U-rich (2/8 bases are G/C); however, miR-21 is not believed to have high target abundance by our calculation (Fig. 2C) or the Bartel group's (39th percentile compared to the 90th percentile for miR-23). As a result, Context+ consistently ranked $\mathrm{miR}$ 21 targets higher than those of miR-23 but often lower than those of miR-122 or let-7 (Fig. 2D). In relation to our comparison between array profiling results and target predictions, the mean scores for the top 30 Context+ predictions were not significantly different for miR-21 and miR-122 targets (Fig. 2E), yet predictions for miR-122 were far more accurate (Fig. 2A). Therefore, we conclude that miR-21, like miR-23 and lsy-6, binds targets with unusually low thermodynamic stability, possibly explaining its substandard repression proficiency. Still, the exhibited activity of miR-21 is not entirely explained by the most recent predictive models nor is it completely analogous to miR-23 or lsy- 6 as it has comparably few target sites.

We also compared our experimental results with known targets of each miRNA, supported by experimental evidence in mice or human samples and curated in the miRecords database of validated miRNA:target associations (Xiao et al. 2009). We found only $12.5 \%$ of all validated miR-21 targets to be significantly up-regulated upon anti-miR treatment, with the prominent miR-21 targets Pdcd4, Pten, and Reck among the majority of targets that were not up-regulated (Fig. $2 \mathrm{~F})$. In comparison, miR-122 and let-7 inhibition derepressed $90 \%$ and $15.8 \%$ of all previously reported targets, respectively. The discrepancy between miR-122 and let-7 was unexpected considering 
the overall strong repression signatures and agreement with prediction algorithms found for these two miRNAs. We note, however, that many of the curated targets for miR-122 are derived from a single study that used antimiRs to inhibit liver miR-122 (Elmén et al. 2008), similar to our own, which explains the consistency. In contrast, the curated targets for let-7 are individually referenced from a number of different studies from a wide range of biological samples. These observations emphasize the importance of biological context when assessing specific miRNA-target pairings.

\section{Stress response genes are exceptionally sensitive to miR-21 inhibition}

Since the limited transcriptome response to miR-21 inhibition corresponded well with neither miRNA target predictions nor previously reported targets, we next turned to gene ontology classifications to identify whether a biological relationship existed among the up-regulated genes. Closer inspection showed that the most up-regulated genes induced by anti-miR-21 treatment belonged to the DnaJ family of co-chaperones, with Dnajal as the single most up-regulated $8 \mathrm{~m}$-seed-matched target, and heat shock proteins, including several $\mathrm{Hsp} 90$ variants and $\mathrm{Hsph} 1$, as the most up-regulated genes in the entire profiling data set (Fig. 3A). Interestingly, stress response genes were significantly enriched $(P<0.05$, binomial test) (Thomas et al. $2003,2006)$ only in both the anti-miR-21 up-regulated seed-matched $(P=0.028)$ and nonmatched $(P=0.018)$ data sets. The large majority of these genes were not significantly up-regulated by the other anti-miRs, disfavoring the notion that the stress response pathway may have been triggered by a nonspecific effect of the anti-miR treatment (Fig. 3A). Many of the activated stress response genes do not contain a miR-21 seed-match, perhaps indicating that they are up-regulated due to derepression of a transcription factor (Fig. 3A, nonseed containing genes are marked in black). Notably, stress response proteins are coordinately transcribed by heat shock factors (HSFs), which trimerize in response to stress and activate transcription by releasing RNA polymerase II (pol II) from a TATA binding protein (TBP)-dependent TFIID transcription initiation complex, stalled at the promoter regions of DNA loci encoding chaperones and co-chaperones (Yuan and Gurley 2000; Akerfelt et al. 2010). Although we did not find an upregulation of HSFs (Fig. 3B), we did, however, discover that several TBP-associated factors (TAFs) were strongly upregulated, particularly Taf7, which contains a miR-21 $8 \mathrm{~m}$ seed in its $3^{\prime}$ UTR (Fig. 3B). Recombinant Taf7 was previously found to associate with the HSF1 oligomerization domain in vitro, and some models suggest an active role for TAF proteins in HSF transcriptional activation (Yuan and Gurley 2000). Thus, it emerges that miR-21 may regulate heat stress response cascades, possibly by directly re-

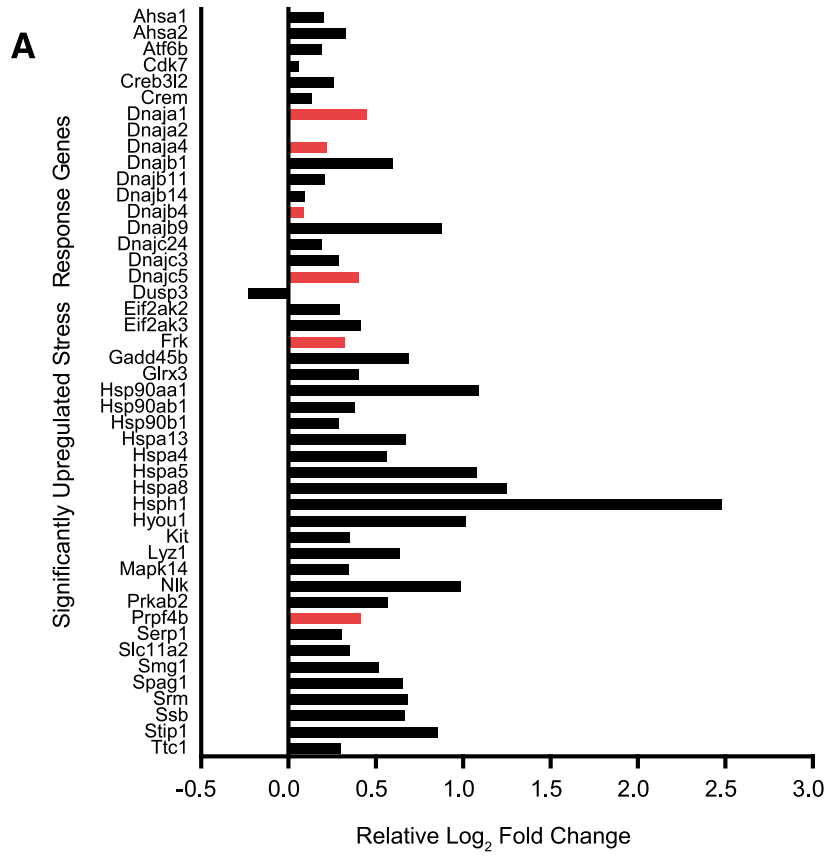

B

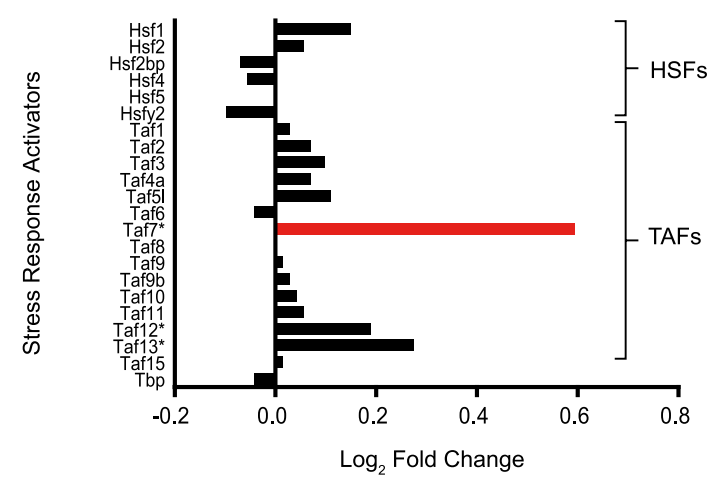

FIGURE 3. miR-21 inhibition induces expression of stress response genes. (A) mRNA changes for significantly up-regulated stress-response genes following anti-miR-21 treatment. Fold-change levels are plotted relative to those observed for the other anti-miR treatments [fold-change anti-21 - mean fold-change $\left.{ }_{(\text {anti-122, anti-let-7) }}\right]$ to demonstrate the anti-miR-sequence dependence of induction. Red bars indicate that the gene transcript contains a seed match for miR-21. (B) mRNA changes for stress response activators (HSFs) or related transcriptional genes (TAFs and TBP). Fold-change levels represent those observed for anti-miR-21 treatment alone. A star marks each gene that was significantly up-regulated upon miR-21 inhibition. Red bars indicate that the gene contains a seed match for miR-21.

pressing Taf7, although we cannot rule out alternative mechanisms.

\section{miR-21 is sparsely associated with translating polysomes}

We next sought to investigate the functional basis for miR21 's diminished silencing activity. We hypothesized that 
miR-21 may be less associated with mRNA due to either weak target-binding or assembly of miR-21 into alternative RNAprotein complexes that prevent it from engaging target transcripts. To assess this possibility, we performed polysome analysis on linear sucrose gradients and compared the distribution of miR-21 to those of miR-122 and let-7. Such analyses have shown that miRNAs typically fractionate predominantly with translating ribosomes, likely by base-pairing with target mRNAs (Kim et al. 2004; Nelson et al. 2004; Maroney et al. 2006; Nottrott et al. 2006). Cytoplasmic lysates prepared from perfused liver tissue in a manner that preserves the majority of all tested miRNAs (Supplemental Fig. S3) were fractionated by centrifugation on $20 \%-60 \%$ sucrose gradients. Analysis of the ribosomal RNA (rRNA) content of each fraction based on absorbance at $\lambda=260 \mathrm{~nm}\left(\mathrm{~A}_{260}\right)$ and electrophoresis of total RNA on denaturing agarose gels revealed that polysomes, representing mRNAs associated with multiple ribosomes, sedimented into the densest half of the gradient (fractions 11-20) (Fig. 4A), as expected. We note that cycloheximide as an inhibitor of translation was not necessary to prevent runoff under our conditions, and its inclusion did not have an effect (Supplemental Fig. S4); we, therefore, did not typically include it in our assays. Western blots showed that the essential RISC proteins Argonaute 2 (Ago2) and TARRNA binding protein (Trbp) (MacRae et al. 2008) were present throughout the gradient, with the most significant proportion found in fractions $1-4$ at the top of the gradient (Fig. 4A). Using real-time quantitative PCR (RT-qPCR), we analyzed the abundance of miRNAs in each fraction and found that miR-21 displays a significantly different distribution compared to miR-122 and let$7 \mathrm{~d}$, a representative isoform of the let-7 family (Fig. 4B). While $\sim 50 \%$ of both miR-122 and let-7d were detected in the polysome-containing fractions, miR-21 was $\sim 2.5$-fold depleted in these fractions (Fig. 4C) but enriched by a similar margin in the top fractions of the gradient where RISC proteins are most abundant. These results appear to be independent of overall

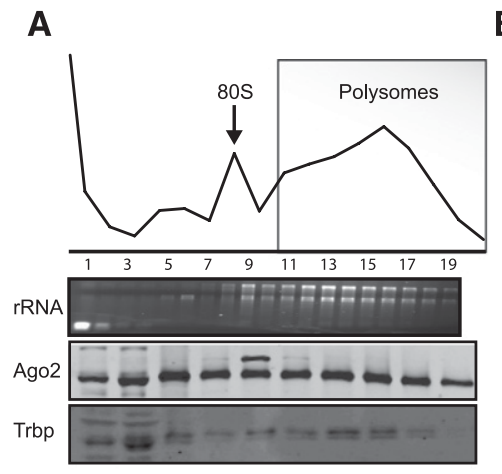

B
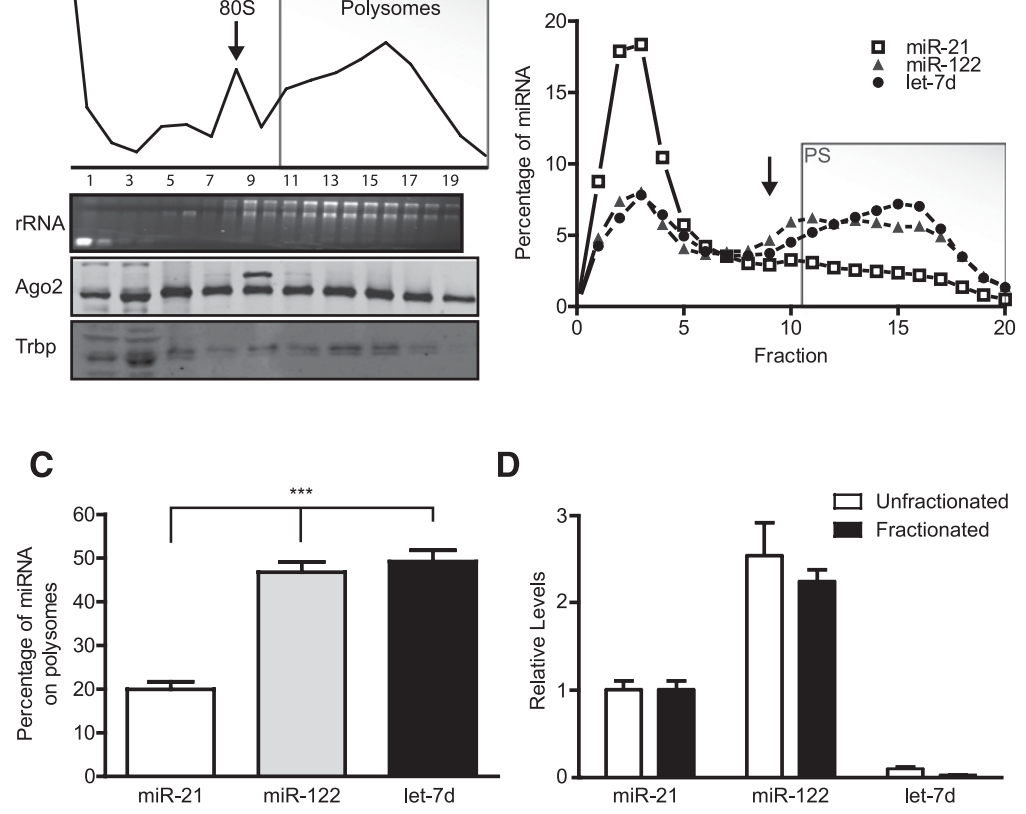

D
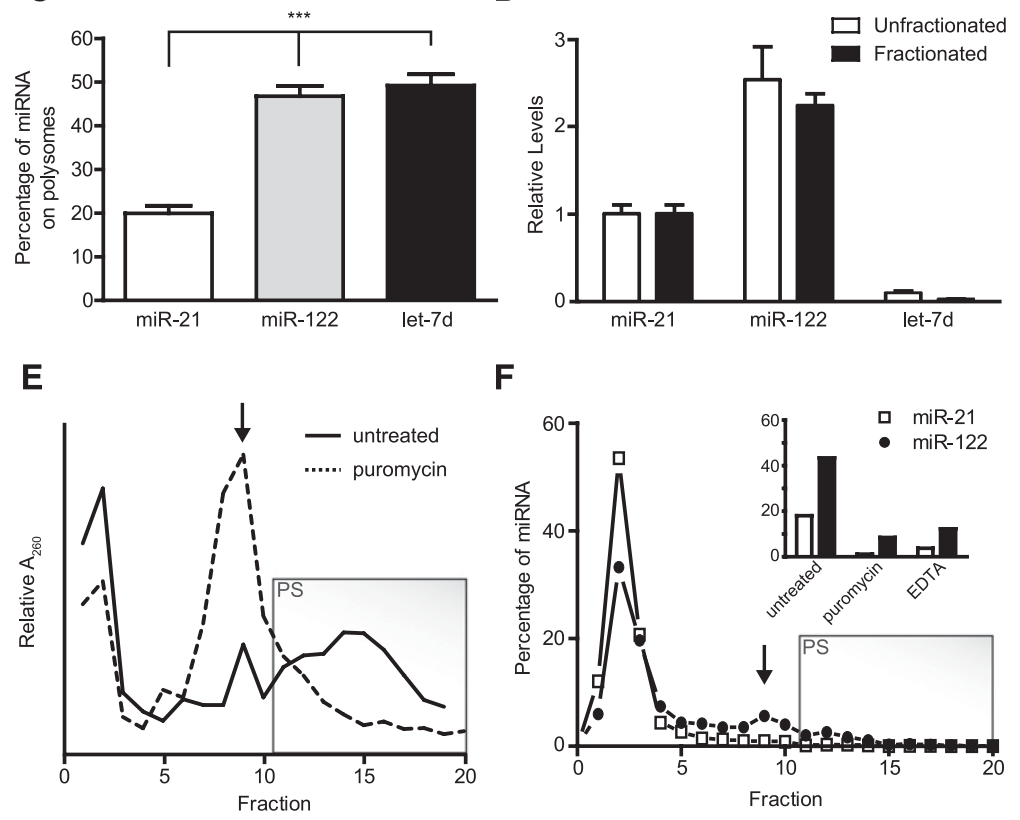

$\mathbf{F}$

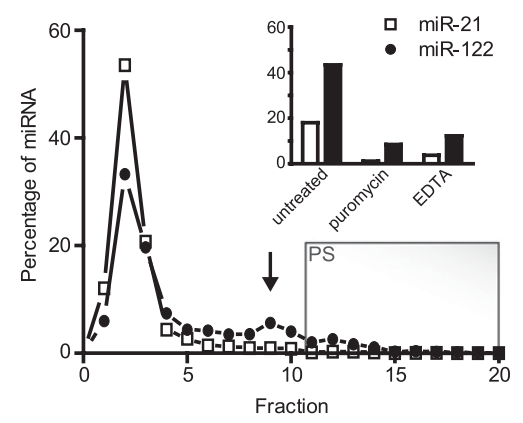

FIGURE 4. miR-21 is disproportionally lacking in polysomal complexes. (A) Top, A260 profile and denaturing agarose analysis of sucrose gradient fractions. Arrow indicates the $80 \mathrm{~S}$ fraction, and the gray box indicates the fractions containing polysomes (PS). Bottom, Western blots of pooled adjacent fractions confirming the presence of RISC proteins throughout the gradient. Inputs for lanes 3-10 were concentrated 10 -fold by precipitation prior to loading, while lanes 1-2 were not further concentrated. $(B)$ Equal volumes of purified total RNA from each fraction were analyzed with RT-qPCR for the presence of miR-21 (white square), miR122 (gray triangle), and let-7d (black circle). The fraction copy number for each miRNA is plotted as the percent total copy number detected from all fractions. Each data point shown is the mean from a total $N=7$ from three independent experiments. $(C)$ The mean summed percent total from $B$ in fractions 11-20 (highlighted with gray box) for miR-21 (white bar), miR-122 (gray bar), and let-7d (black bar). $\left.{ }^{* * *}\right) P<0.001$, calculated by one-way analysis of variance (ANOVA) with Bonferroni post-test. $(D)$ Relative miRNA levels before (unfractionated; white bar) or summed after (fractionated; black bar) sucrose gradient fractionation. Error bars represent standard error of the mean (SEM). (E) miRNA sedimentation is sensitive to translational drop-off. Lysates were treated with puromycin or EDTA to disrupt translation. $\mathrm{A}_{260}$ profiles for puromycin-treated (dashed line) and untreated (solid line) samples. $(F)$ miRNA distributions resulting from puromycin treatment. Inset, comparison of miR-21 (white bars) and miR-122 (black bars) summed percent totals in the densest fractions (normally taken to be polysomecontaining fractions) for untreated, puromycin-, and EDTA-treated samples.

miRNA abundance since the relative levels of miR-122 and let-7d are greater and less than that of miR-21, respectively (Fig. 4D). 
The association of miRNAs with polysomes was previously reported for cultured HeLa cells (Maroney et al. 2006; Nottrott et al. 2006). To confirm that these results are consistent in vivo and that cosedimentation was not coincidental, we tested whether the distribution of miRNAs in polysome-containing fractions could be disrupted with puromycin, a tRNA-mimic that blocks elongation of actively translating complexes. As expected, treatment of lysates with puromycin prior to loading on sucrose gradients caused a loss in polysomes and a resulting increase in $80 \mathrm{~S}$ ribosomes (Fig. 4E). miRNAs showed a similar shift away from the densest fractions toward the top of the gradient (Fig. 4F). In addition, we tested the effect of EDTA, which causes dissociation of polysomes and monosomes into 40 S and 60 S ribosomal subunits (Supplemental Fig. S5). Again, polysome dissociation was matched by the loss of miRNA sedimentation into the densest fractions (Supplemental Fig. S5; quantified in Fig. 4F, inset). Interestingly, miR-122 under these conditions still sedimented with the $80 \mathrm{~S}$ ribosome fractions, perhaps due to the presence of a RISC-loading complex of similar sedimentation properties (Pham et al. 2004).

Taken together, these results suggest that, in healthy mouse liver, miR-21 is associated with actively translating complexes to a significantly lesser extent than other miRNAs.

\section{miR-21 is able to bind complementary RNA and remains unassociated with polysomes upon administration of its anti-miR}

To further probe for a correlation between miR-21's altered subcellular distribution and its mRNA silencing activity, we analyzed miRNA gradient distributions following anti-miR treatment. Mice were treated with a single dose of anti-miR $24 \mathrm{~h}$ prior to gradient analysis. This quick treatment, sufficient to elicit a measurable response using Sylamer analysis for anti-miR-122, manifested in the gradients as a nearly complete shift of miR-122 away from all but the lowest-density fractions (Fig. 5A). In polysome-containing fractions, miR-122 enrichment was reduced $\sim 20$-fold with
A

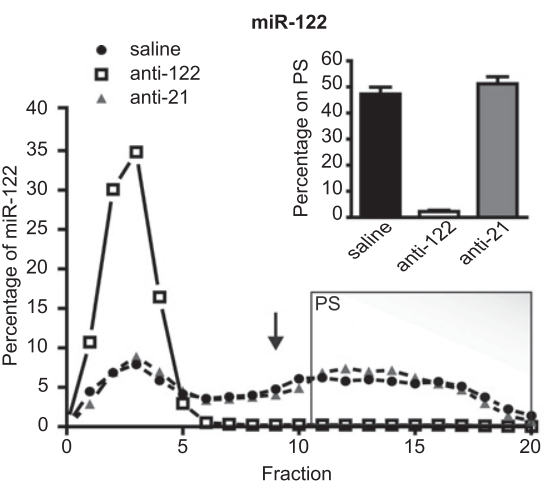

$\mathbf{B}$

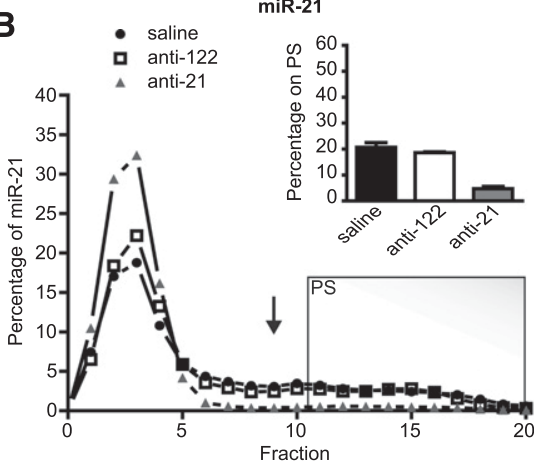

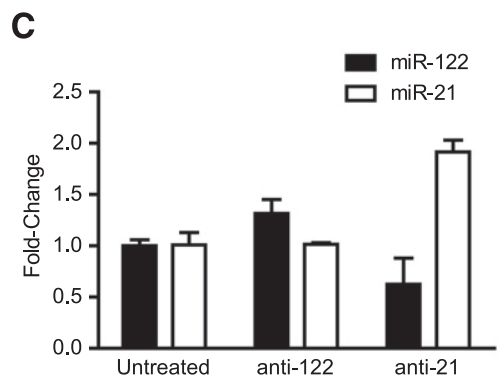

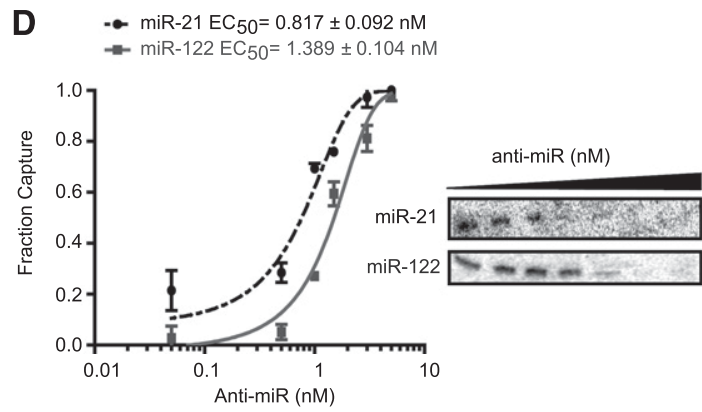

FIGURE 5. The subcellular distribution of miR-21 resembles that of inhibited miRNA. (A) Percent distribution profile of miR-122 in sucrose gradients following a single dose of saline (black circle or bar), anti-122 (white square or bar), or anti-21 (gray triangle or bar) $24 \mathrm{~h}$ prior to harvest. Arrow indicates the $80 \mathrm{~S}$ fraction, and the gray box indicates the fractions containing polysomes (PS). Inset, the corresponding mean summed percent total in polysomal fractions for each treatment. (B) As in $A$ for miR-21. (C) Comparison of miRNA levels in anti-miR treated lysates prior to gradient fractionation. The relative miR-122 (black bars) and miR-21 (white bars) levels were calculated with the $2^{-\Delta \Delta \mathrm{Ct}}$ method (Schmittgen and Livak 2008) using miR-22 as a reference. The normalized fold-change levels of miRNA were further set to 1.0 in saline-treated animals. $(D)$ miRNA binding assay with 2'-O-methyl complementary capture RNA. Biotinylated 2'-O-methyl oligos complementary to either miR-21 (black circle and dashed line) or miR-122 (gray square and solid line) were titrated into S16 liver extracts. Bound miRNA was depleted from the extract by precipitation of the capture RNA using streptavidin-coated beads, while unbound miRNA was detected by Northern blot (shown here from a representative experiment). Data were fit with a sigmoidal dose-response curve with variable slope (for miR-21: $\mathrm{K}_{1 / 2}=0.817$ $+/-0.092 \mathrm{nM}$, hill-slope $=1.080+/-0.2194, \mathrm{R}^{2}=0.962$; for miR-122: $\mathrm{K}_{1 / 2}=1.389+/-0.104 \mathrm{nM}$, hill-slope $\left.=1.130+/-0.312, \mathrm{R}^{2}=0.967\right)$. Data points were averaged from duplicate experiments, and error bars represent SEM. 
anti-miR-122 compared to saline and anti-miR-21 treated animals (Fig. 5A, inset). This shift was sequence-selective, thus is very likely due to complementary base-pairing between anti-miR and miRNA. This observation suggests that miRNAs sediment with polysomes because they are directly bound to mRNAs rather than associated with ribosomes through interactions bridged by RISC components.

While miR-21 showed a similar shift to low-density fractions upon treatment with anti-miR-21, the effect was significantly minimized due to the fact that miR-21 was already preferentially distributed in the low-density fractions even in the absence of anti-miR (Fig. 5B). As a result, anti-miR-21 induced only an approximately fourfold change of miR-21 in the polysomal fraction, about fivefold less of an effect than that seen with miR-122 and anti-miR-122 (Fig. $5 B$, inset).

Despite reports to the contrary, anti-miRs do not cause degradation of cognate miRNAs; they can, however, interfere with miRNA detection, making it appear as if the miRNA has been degraded (Davis et al. 2009). To ensure that the observed anti-miR-induced shifts were genuine and not a detection artifact, the relative levels of miRNAs in treated and untreated lysates were compared prior to loading on gradients. Levels of miR-122 and miR-21 were not reduced following anti-miR treatment (Fig. 5C), indicating that under our experimental conditions, anti-miR did not interfere with RT-qPCR measurements. Surprisingly, miR-21 instead showed about a twofold increase in expression with anti-miR-21 treatment (Fig. 5C). This observation may be attributed to either release of otherwise immeasurable miR-21 from sequestered populations or de novo miR-21 expression triggered by a feedback loop in response to anti-miR mediated inhibition, perhaps suggesting that minimal silencing activity of miR-21 is still relevant to signaling pathways (see Discussion). Still, even with such increased levels, we infer that miR-21 is fully inhibited by anti-miR based on the extent of depletion from dense fractions of the sucrose gradient (Fig. 5B).

Since the anti-miR inhibited miR-21 resembles the uninhibited miRNA on the sucrose gradient, we investigated whether miR-21 could at all bind complementary RNA. Liver lysates were prepared as usual and incubated with increasing concentrations of $5^{\prime}$-biotin-end-labeled 2'-Omethyl oligonucleotides fully complementary to either miR122 or miR-21. After pull-down with streptavidin-coated beads, the depletion of each miRNA was quantified using Northern blot. The entirety of measurable miR-21 was depleted with a half-titration point of $\mathrm{K}_{1 / 2}=0.82 \pm 0.09$ $\mathrm{nM}$, while miR-122 was depleted with a $\mathrm{K}_{1 / 2}=1.4 \pm 0.1 \mathrm{nM}$ (Fig. 5D). Considering that miR-122 levels in our lysates are $\sim 2.5$-fold those of miR-21 (Fig. 4D), the binding results indicate that miR-21 is, in principle, capable of binding a complementary RNA just as efficiently as miR-122.

The findings that systemically delivered anti-miRs cause an up-regulation of corresponding mRNA targets and block the binding of their respective miRNAs to polysome-bound mRNAs indicates that the miRNA-polysome interaction is critical for miRNA-mediated silencing. Consequently, the severely reduced binding of miR-21 to polysomes is consistent with its decreased mRNA silencing activity and the reduced response of its mRNA targets to the administration of anti-miR-21.

\section{A known target mRNA is largely inaccessible to $\mathrm{miR}-21$}

Our interpretation that intracellular access and binding of miRNA to polysomes is important to miRNA-mediated silencing assumes that repressed transcripts are, indeed, associated with polysomes. While mRNA is necessary for polysome formation, previous cell culture studies have shown that some mRNA targets are not associated with polysomes during repression by miRNA (Pillai et al. 2005; Bhattacharyya et al. 2006). Instead, these repressed mRNAs are translationally incompetent and sediment in low-density fractions of sucrose gradients.

To determine the location of repressed and nonrepressed mRNAs under our experimental conditions, we measured the distribution of AldoA and Pdcd 4 mRNA in the presence and absence of anti-miR. These mRNAs are previously validated targets of miR-122 and miR-21, respectively. Consistent with our profiling data, RT-qPCR showed a fourfold derepression (increase) of AldoA mRNA levels upon anti-miR-122 administration, confirming it as a miR122 target. In contrast, we found no changes in Pdcd4 levels upon treatment with anti-miR-21, indicating the absence of a regulatory interaction with miR-21 (Fig. 6A). Unlike observation during the earlier cell culture studies (Pillai et al. 2005; Bhattacharyya et al. 2006), the largest fraction of both mRNAs $(\sim 80 \%)$ was found in polysomes under salinetreated conditions, demonstrating that repression by endogenous liver miRNAs does not prevent (at least partial) translation of target mRNAs (Fig. 6B,C). In the presence of the corresponding anti-miR, both AldoA and Pdcd4 mRNAs shift slightly from fractions 16-20 to fractions 10-14; however, this shift does not significantly reduce the total percentage of mRNA contained in polysomal fractions (Fig. 6D). This finding indicates that anti-miRs can cause slight changes to translation in a sequence-independent manner reminiscent of a mild interferon-like response (Manche et al. 1992; Reynolds et al. 2006), rather than the miRNAmediated effect observed with miR-122 in Huh-7 cells (Bhattacharyya et al. 2006) and with let-7 in HeLa cells (Pillai et al. 2005).

Three Pdcd4 transcript variants are recorded in the NCBI Reference Sequence (RefSeq) database. All three are transcribed from the same locus and code for the same protein; however, the mRNAs differ in $5^{\prime}$ and $3^{\prime}$ UTR length (Fig. 6E, left). While variant 1 (NM_011050.4) and variant 2 (NM_001168491.1) have identical 3' UTRs, and both contain 
A
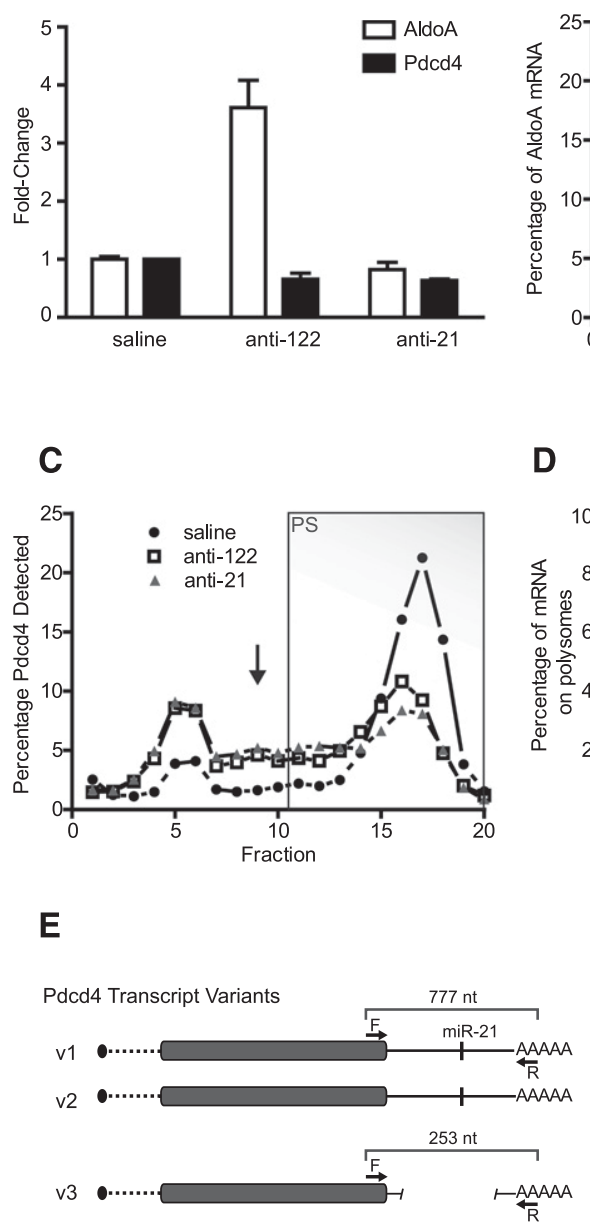

B

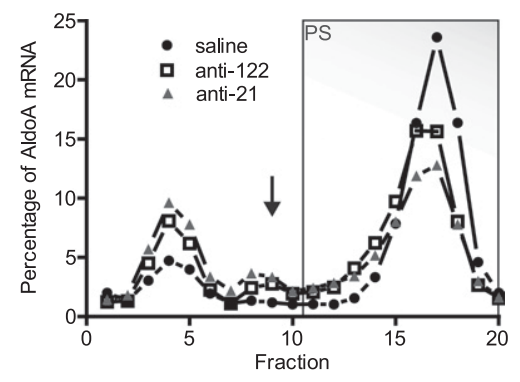

D
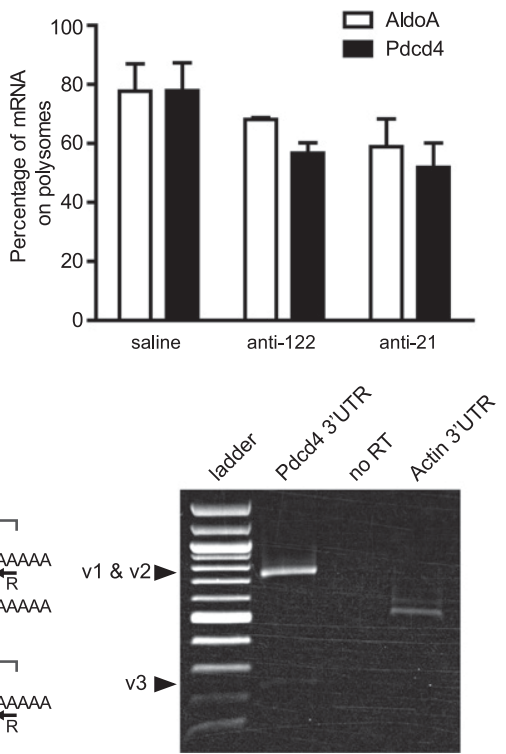

FIGURE 6. Predicted mRNA targets remain associated with polysomes in the presence and absence of miRNA-mediated repression. (A) Relative levels of AldoA (white bar) and Pdcd4 (black bar), following treatment with anti-miR. mRNA levels were calculated with the $2^{-\Delta \Delta \mathrm{Ct}}$ method using GAPDH as a reference. $(B)$ Percent distribution profile for AldoA mRNA, a predicted target for miR-122, in sucrose gradients following a single dose of saline (black circle), anti-122 (white square), or anti-21 (gray triangle) $24 \mathrm{~h}$ prior to harvest. Arrow indicates the $80 \mathrm{~S}$ fraction, and the gray box indicates the fractions containing polysomes (PS). (C) As in $B$ for Pdcd4. $(D)$ The corresponding mean summed percent totals of AldoA (white bars) and Pdcd4 (black bars) mRNA in polysomal fractions for each treatment. Data points were averaged from duplicate experiments, and error bars represent SEM. (E) Three variants of the Pdcd4 transcript are encoded in the same locus in the mouse genome. Left, schematic of variant sequence alignment. The transcripts, which code for the same protein, have variable $5^{\prime}$ UTRs and identical open reading frames (gray box) and 3' UTRs, except for variant 3 , where the miR-21 binding site is absent, but the upstream and downstream portions of the $3^{\prime}$ UTR are retained. Arrows mark the sites for forward (F) and reverse (R) primers for sequence length determination using $3^{\prime}$ rapid amplification of cDNA ends (RACE). Right, agarose gel of PCR products from 3' RACE. The expected lengths for the variants are marked by arrowheads. Based on intensity comparison between the slower migrating and faster migrating bands, the larger variants containing miR-21 seed matches make up $\geq 90 \%$ of the Pdcd 4 transcript population. Reactions containing no reverse transcriptase (no RT) or primers for the actin $3^{\prime}$ UTR served as negative and positive controls, respectively.

a single miR-21 8m seed-match, variant 3 (NM_001168492.1) does not contain a seed-match due to the absence of an $\sim 500$ nt segment flanking the miR-21 site. Intriguingly, the remainder of the 3' UTR, both upstream of and downstream from the miR-21 site, is identical to that of the other variants, suggesting that variant 3 may have evolved to avoid miR-21 regulation. If variant 3 were the predominant transcript in mouse liver, this would explain why we did not observe derepression of this highly referenced target. To test the relative abundance of the variants, $3^{\prime}$ rapid amplification of cDNA ends ( $3^{\prime}$ RACE) was employed to amplify the $3^{\prime}$ UTRs of transcripts containing the Pdcd4 open reading frame from total RNA. Agarose gel electrophoresis of the amplified product displayed two bands with lengths of $\sim 750$ bp and $\sim 250 \mathrm{bp}$, which matched the expected lengths of variants $1 / 2$ and variant 3, respectively (Fig. 6E, right). Densitometric analysis of the relative band intensities showed $\geq 90 \%$ of the Pdcd4 transcript population to be of the longer variety, indicating that the majority of Pdcd4 expressed in mouse liver contains a miR-21 binding site.

We conclude that mRNA targets of miRNAs retain translational capacity and reside in polysomes, regardless of the extent of their regulation. Taken together with miR-21's observed subcellular distribution in healthy liver, a known target mRNA such as Pdcd4 appears predominantly inaccessible to the abundant intracellular miR-21.

\section{In cancer cells, miR-21 is highly associated with polysomes and has a strong repression signature}

In notable contrast to our findings in liver, Nilsen and colleagues have previously reported miR-21 as highly associated with polysomes in cervical adenocarcinoma HeLa cells (Maroney et al. 2006), where measurable miR-21 target repression is observed (Yao et al. 2009). To verify these results, HeLa cell extracts were prepared as described by these authors, analyzed on sucrose gradients, and miR-21 was quantified by RT-qPCR. Consistent with the previous report, $\sim 60 \%$ of miR-21 was distributed in polysome-containing fractions in similar abundance as that observed for liver let-7 and miR-122 (Fig. 7A). Additionally, let-7a was also found to have enhanced polysome association in HeLa cells when compared to liver, with nearly all let-7a detected in polysomal fractions (Fig. 7A). This observation suggests that miRNA 
A

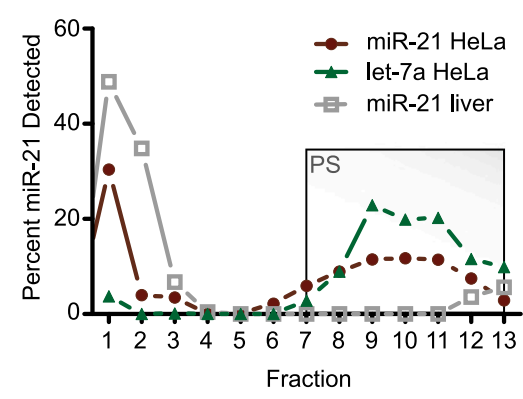

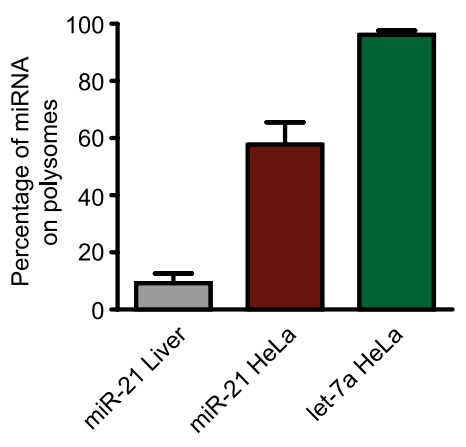

B

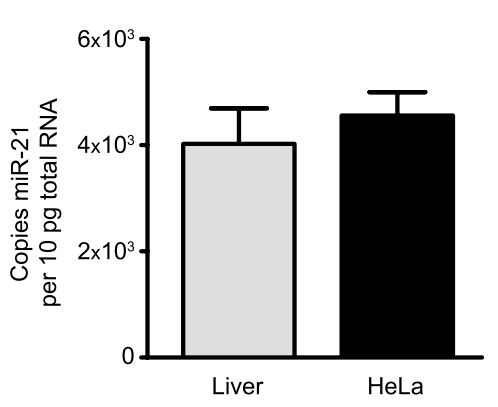

C

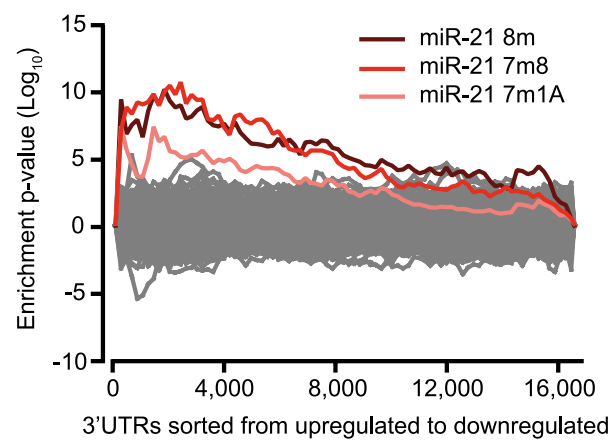

D

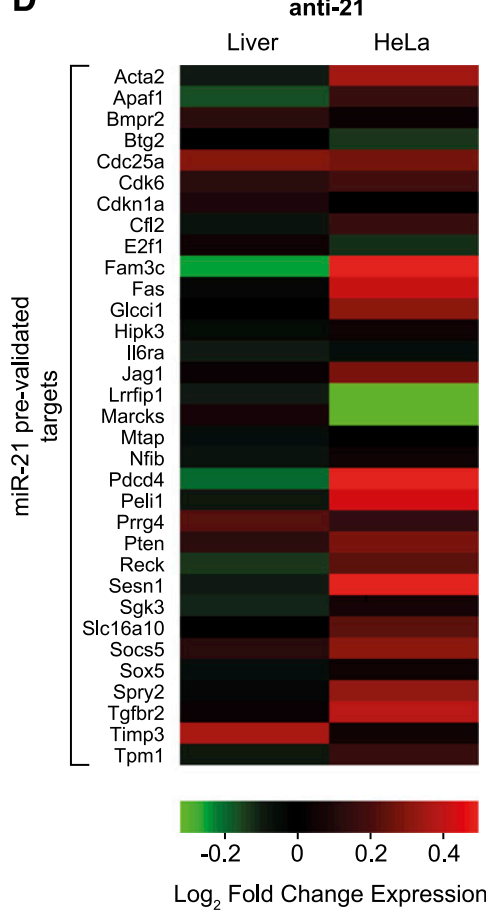

FIGURE 7. miR-21 is highly associated with polysomes and strongly represses a broad range of targets in HeLa cells. (A) Left, percent distribution profile of HeLa miR-21 (dark red circles), HeLa let-7a (dark green triangles), and liver miR-21 (gray open squares) in sucrose gradients loaded with mouse liver or HeLa extracts prepared under the same conditions. The gray box indicates the fractions containing polysomes (PS). Right, the mean summed percent total in polysome fractions 7-13 (highlighted with gray box) for each miRNA (colors are the same as in the left panel). Error bars represent SEM from triplicate experiments. (B) Absolute quantification of miR-21 copy numbers in liver and HeLa lysates normalized to total input RNA. Error bars represent SEM from $N=4$ (HeLa) and $N=3$ (liver) biological replicates assayed in two independent experiments. $(C)$ Heptanucleotide Sylamer analysis of array profiling of HeLa cells transfected with anti-miR-21 (compared to saline mock transfection). The seed matches for each miRNA are highlighted. All other possible 7-nt sequences not related to the seed match are shown as gray lines and thus represent statistical background noise. The $8 \mathrm{~m}$ seed match from the octanucleotide analysis is shown superimposed. Heptanucleotide and octanucleotide analyses had similar backgrounds. The peaking of enrichment for miR-21 seed-matched sequences on the left-hand side of the plot indicates that the genes most up-regulated upon miRNA inhibition are enriched for the corresponding seed-matched sequence (cf. mmu liver in Fig. 1D, bottom, left). (D) Heat map comparison of responses in mRNA levels for known targets from inhibition of miR-21 in mouse liver or HeLa.

target engagement may be globally enhanced in HeLa cells. As a control, only $\sim 12 \%$ of liver miR-21 from gradients prepared using identical conditions were found in polysome fractions, even less than using our original preparation conditions (cf. Fig. 7A and Fig. 4B,C).

Elevated levels of miR-21 are detected in both liver and cervical cancer cell lines relative to their corresponding healthy source tissues (Lui et al. 2007; Meng et al. 2007). To determine whether a higher abundance of miRNA was responsible for the observed increase in polysome binding, we measured miR-21 copy numbers per amount of total input RNA isolated from liver or HeLa cells. Coincidentally, both samples contained comparable levels of miR-21 at 40005000 copies per 10 pg input RNA (Fig. 7B). We conclude that miR-21's distinct polysome binding profile in liver and HeLa cells is not due to differences in expression levels.

Next, we investigated whether this increased association with polysome-bound mRNA translated to a stronger repression profile. HeLa cells transfected with either anti-miR-21 or saline were array profiled and analyzed with the Sylamer algorithm (Fig. 7C). Contrasting with our findings for liver miR-21, inhibition of HeLa miR-21 resulted in a measurable derepression of mRNAs enriched with 7- or 8-nt seed matches. In addition, many of the previously validated targets that were not depressed in liver, such as Pdcd4, were strongly derepressed in HeLa (Fig. 7D).

These findings support the conclusion that polysome complexes are important for miRNA-mediated repression in cancer cells, as increased repression by miR-21 is associated with increased binding to polysome-bound mRNA. Our HeLa cell results are thus consistent with previous observations (Kim et al. 2004; Nelson et al. 2004; Maroney et al. 2006; Nottrott et al. 2006) and further underscore the unusually limited RNA silencing activity of the abundant miR-21 in healthy liver. 


\section{DISCUSSION}

In contrast to its oncogenic prowess in cancer cells, miR-21 is a surprisingly weak RNA silencer in healthy liver tissue

In the current study, we have examined the in vivo activity of miRNAs in healthy mouse liver tissue using array profiling and polysome analysis. Although testing of additional tissues and cell lines is desirable, our results strongly suggest that miR-21, relative to other miRNAs, is functionally limited under normal physiological conditions, yet not in cultured cancer cells. Evidence for this conclusion is provided by the following observations: (1) pharmacological inhibition or knockout of miR-21 does not result in an up-regulation of mRNA targets significantly enriched for seed sequence matches; (2) miR-21 exhibits reduced binding to translating target mRNAs, which represents the largest population of an mRNA in the cell; (3) the sucrose gradient distribution of miR-21 alone resembles that of the anti-miR inhibited miRNA, suggesting that miR-21 is impeded from interacting with polysome-bound mRNA equally in the absence as in the presence of its anti-miR. In contrast, by analyzing the global up-regulation of mRNAs following miRNA inhibition, we were able to observe trends for miR122 and let-7 consistent with canonical miRNA-target prediction rules (Grimson et al. 2007). That miR-21 displays these trends so minimally in liver challenges the current assumption that the rules of engagement of highly expressed miRNAs are the same across different tissues and cell types. These results may extend to other healthy tissues as well, since previous studies have hinted that miR-21 is also surprisingly underactive in lung and heart in the absence of applied stress (Hatley et al. 2010; Patrick et al. 2010).

Might idiosyncrasies in miR-21's mechanism of silencing explain our results? That miR-21 represses gene output by inhibiting translation directly without causing changes to mRNA levels cannot be unequivocally dismissed; however, we found little evidence to suggest an up-regulation of translational capacity in the absence of miRNA-mediated repression. More so, a translation-based mechanism compared to a degradation-based mechanism arguably would necessitate an even more pronounced interaction of miRNA with translating complexes, which we found to be lacking.

miR-21's meager repression signature in healthy cells is surprising considering its reported oncogenic prowess, capable of inducing the formation of tumors that are dependent solely on its silencing activities (Medina et al. 2010). Considering these apparently contradictory activities, our results suggest that miR-21 becomes "activated" during disease or other aberrant states, broadening both its repression footprint and its impact on the cellular state. In line with this conclusion, we found a large number of seedmatched targets to be derepressed upon inhibition of miR21 in adenocarcinoma HeLa cells. Many of these affected transcripts have been found to be the downstream targets, underpinning miR-21's pathological contribution in tumor modulation (Jazbutyte and Thum 2010), and similar derepression of targets has been reported upon inhibition of miR-21 in MCF-7 breast cancer cells (Frankel et al. 2008). Correspondingly, we observed starkly different subcellular locations of miR-21 under the two biological contexts: miR21 in mouse liver predominantly sediments in light fractions apart from mRNAs, while in HeLa lysates, miR-21 predominantly sediments in polysomal fractions with mRNA. Cosedimentation of miRNA/mRNA was not coincidental, as it was dependent on miRNA base-pairing and was sensitive to small-molecule translational inhibitors. Therefore, we posit that miR-21's increased repression proficiency in HeLa is at least partly due to enhanced binding to its targets. However, the mechanism(s) underlying this enhancement in HeLa cells-or alternatively, the diminished activity in liver tissue-are still unclear. Considering that let-7a also shows additional polysomal binding in HeLa cells suggests that this enhanced target loading capacity may be due to an increased abundance of a common protein factor like the receptor for activated C-kinase (Rack1), a 40S ribosomal protein and essential component for miRNAmediated repression involved in loading miRISC onto translating mRNAs (Jannot et al. 2011a).

\section{What may cause miR-21's limited RNA silencing activity in healthy liver?}

It is conceivable that miR-21's reduced binding and repression in liver tissue are attributable to thermodynamics. Indeed, computational analysis predicted miR-21 to bind targets less stably than other miRNAs largely due to high $\mathrm{A}+\mathrm{U}$ richness in the seed region. Binding assays showed that miR-21 binds a complementary 2 '-O-methylated RNA as effectively as does miR-122; however, these assays merely demonstrate miR-21's potential to bind target RNAs. The thermodynamics of miRNA:mRNA binding are not reflected due to the unusually high extent of base-pairing and the $2^{\prime}$ O-methyl modifications of the complementary RNA, which both raise the free energy of duplex binding (Majlessi et al. 1998). Interestingly, miR-23 and lsy-6, miRNAs also with $\mathrm{A}+\mathrm{U}$ rich seeds, have similarly been reported to have limited repressive activity (Garcia et al. 2011). However, unlike miR21 , these miRNAs additionally have high target abundance that further dilutes their effects on the transcriptome. Although experimental work demonstrated that these properties-weak seed pairing and high target abundance - can independently contribute to diminished proficiency, it is uncertain whether weak seed pairing alone could explain our observations for liver miR-21. The current "Context+" predictive model, which takes these properties into account, suggests not, since the top predicted targets of miR-21 were scored equally high as those of miR-122, indicating that miR-21 is still predicted to repress targets similarly to miR-122, despite its weaker 
seed pairing. It should be noted, however, that the Context+ algorithm and its "Context only" predecessor were both trained with array profiling experiments from HeLa cell culture. Accordingly, and consistent with our own HeLa cell data, these algorithms score miR-21 targets highly. These considerations lead us to propose a model wherein miR-21 in liver tissue, despite its abundance, is expressed at a level below the threshold required to bind and repress a wide range of targets in this cellular environment. Interestingly, the same expression level is sufficient to elicit target repression in HeLa, which indicates that these cells are generally more conducive to miR-21 activity and perhaps that of other miRNAs as well. This favorable environment for miRNA-mediated repression may be due to increased availability of pathway components, alterations in the transcriptome landscape, or changes in the activity of other regulatory proteins (e.g., A/U-rich element RNA-binding proteins) that may compete with miRNAs for target binding. However, further investigation will be required to determine whether an increased abundance of miR-21, which frequently occurs under pathological conditions like liver and cervical cancer (Lui et al. 2007; Meng et al. 2007), is alone sufficient to activate target repression. This can be tested in the future by experiments similar to those shown here but using transgenic mice overexpressing liver miR-21.

Additionally or alternatively, miR-21's target abundance may be underestimated. Although the number of different transcripts containing miR-21 target sites is less than those for miR-122 or let-7, the abundances of these transcripts were not factored into our calculation. It is possible that miR-21 targets are expressed to higher levels, causing miR21 's effect on any one gene to be weakened. Yet, such a notion is inconsistent with the observed dominant localization of miR-21 in nonpolysomal fractions, as increased target abundance would be expected to lead to greater relative miR-21 fractionation into polysomes due to increased mRNA binding.

Another possibility is that only specific abundant, yet translationally inactive, transcripts play a role in miR-21's low silencing activity in liver. An increasing amount of recent research has concluded that transcripts of pseudogenes, evolutionarily miscopied gene duplicates that do not code for functional proteins, can strongly influence miRNAmediated gene regulation by sequestering miRNAs away from real targets (Poliseno et al. 2010; Cesana et al. 2011; Sumazin et al. 2011; Tay et al. 2011). For example, the pseudogene PTENP1 can indirectly control expression of its ancestral gene PTEN by "sponging up" miRNAs that bind both transcripts; in this way, increases in the pseudogene's noncoding RNA levels cause increases in the ancestral gene's mRNA levels and vice versa. Since pseudogenes often contain nonsense mutations near the start of the open reading frame, these transcripts would be expected to largely reside in nonpolysomal fractions, possibly explaining miR-21's localization if it were bound to a pseudo- gene(s) (Poliseno et al. 2010; Pink et al. 2011). Intriguingly, miR-21 is presumed to bind PTENP1 in human cells (Pink et al. 2011). Although a Ptenp1 mouse homolog has not been discovered to our knowledge, there remains the possibility that other yet-to-be identified miR-21 binding pseudotranscripts exist. The high sequence similarity between pseudogenes and their ancestral genes complicates a characterization of a large fraction of the pseudotranscriptome by conventional means (i.e., by RT-qPCR and microarrays) (Harper et al. 2003; Pink et al. 2011). The advent of deep-sequencing technology should rapidly enable the measurement of the presence and prevalence of pseudotranscripts and contribute to our understanding of how this endogenous network of miRNA-sponges affects miRNAs such as miR-21.

\section{miR-21 regulation is specifically linked to stress response pathways}

We found a select group of seed-matched targets to be upregulated upon miR-21 inhibition. An interesting question is why these transcripts respond while most do not. Importantly, we found stress response to be a common biological pathway among up-regulated genes both with and without miR-21 seed matches. Weakly binding miRNAs like miR-21 may be particularly suited to regulate response pathways, as they would be more sensitive to subtle transcriptomic fluctuations or other changes in their targets, allowing for a temporal control of miRNA-mediated repression which, in turn, may be responsive to extracellular cues. We also found that miR-21 itself is up-regulated following anti-miR-21 treatment, suggesting the presence of a feedback loop that may act to contain a stress activation event by reestablishing the balance between miR-21 and its targets. Such a feedback loop may explain why miR-21 is overexpressed in chronically stressed, abnormal cell states such as cancer and cardiac disease (Jazbutyte and Thum 2010). We hypothesize that miR-21 exerts its influence on the activation of heat stress response cascades via Taf7, which has been shown to interact with HSF-activation complexes (Yuan and Gurley 2000). Additionally, Taf7 may act as a node for miR-21's integration into additional stress response pathways, as increasing amounts of Taf7 in HEK293 and COS cells correlate with enhanced transactivation of c-Jun, an AP-1 transcription factor activated by stress-activated protein kinases (SAPKs) (Munz et al. 2003; Mehan et al. 2011; Turner 2011). In the future, it will be important to further test for links between miR-21 and stress to determine which stresses and stress-related pathways miR-21 may respond to in various tissues.

Finally, we note that we cannot entirely rule out the possibility that some or all of the seed-matched transcripts, despite containing miR-21 binding sites, are up-regulated as the result of secondary effects of miR-21 inhibition, not because they were directly repressed by miR-21. The current 
standard for validating miRNA:target interactions are reporter gene assays where luciferase constructs are engineered with the 3' UTRs of interest and expressed in cell lines coexpressing the targeting miRNA; selectivity of the interaction is demonstrated if mutation of the seed sequence results in increased luciferase output. However, our findings underscore that assays in cell culture may not be appropriate considering the drastically different miR-21 activities observed between liver tissue and cultured HeLa cells. A better solution in general would, therefore, be to validate target specificity in the same biological context used for the original observation. For example, reporter gene assays mediated by in vitro translation have been previously used to study miRNA activity in Krebs-2 excites extract (Fabian et al. 2011) and rabbit reticulocyte lysate (Wang et al. 2006). Translation-competent liver extracts have been reported (Sampson et al. 1972); thus, further validation using this type of system may be possible for miR-21 in the future.

In conclusion, we find unexpectedly narrow target specificity in healthy liver compared to cancer cells for one of the most abundant, disease-linked miRNAs, demonstrating the importance of biological context in RNA silencing. Future studies will need to test additional tissues and cell lines to confirm the generality of our findings as well as to address the mechanism(s) behind miR-21's regulatory activation in cancer cells in more depth. Focused approaches to compare miR-21 activity in normal and diseased tissue (Hatley et al. 2010; Medina et al. 2010), where miR-21 is cooverexpressed with additional oncogenic or other disease promoting factors, may be particularly powerful to define the conditions required for miR-21 activation.

\section{MATERIALS AND METHODS}

\section{Animal care and treatments}

All animal experiments were conducted according to the Institutional AAALAC Guidelines. Male C57BL/6 mice were housed four to five animals per cage with a $12 \mathrm{~h}$ light/dark cycle. Oligonucleotides were dissolved in saline and administered to mice by intraperitoneal (i.p.) injection. For mRNA profiling studies, mice were treated with $20 \mathrm{mg} / \mathrm{kg}$ twice weekly for $2 \mathrm{wk}$. miR-21 knockout mice were obtained from Dr. Stuart Orkin of Harvard University (Lu et al. 2011).

\section{Cell culture and transfection}

HeLa cells were cultured in DMEM with 10\% FBS. Anti-miR-21 was transfected with Lipofectamine RNAiMax (Life Technology) according to the manufacturer's instructions. For sucrose gradients, HeLa cells were treated with $0.1 \mathrm{mg} / \mathrm{mL}$ cycloheximide 20 min before harvesting. Lysates were prepared by swelling cells on ice in hypotonic buffer $(10 \mathrm{mM} \mathrm{NaCl}, 1.5 \mathrm{mM}$ magnesium acetate, $50 \mathrm{ug} / \mathrm{mL}$ dextran sulfate, $10 \mathrm{mM}$ Tris- $\mathrm{HCl}, \mathrm{pH}$ 7.4) before dounce homogenization. The homogenized lysate was collected, centrifuged at $1200 \mathrm{~g}$ for $5 \mathrm{~min}$ at $4^{\circ} \mathrm{C}$, and loaded on sucrose gradients (see Liver Lysates and Fractionation).

\section{Comparison with computationally predicted targets}

Predicted targets for each miRNA were downloaded from the following databases: miRanda-mirSVR—mouse, good mirSVR score, conserved miRNA, updated August 2010; TargetScan(Conservation) - mouse, conserved targets Pct scores, v5.2; TargetScan(Context)-mouse, conserved targets context scores, v5.2; TargetScan (Context+)-mouse, conserved targets context+ scores, v6.0; microCosm-mouse, v3.0; and PITA-mouse, top predictions, $3 / 15$ flank, v6.0. Starting with the highest score, each set of top predictions was cross-referenced with genes measured by array profiling until the first 30 matches were found. All matches came from, at most, the top 50 predictions from any prediction data set.

\section{Gene ontology classifications and bioinformatic calculations}

For gene ontology assignments, seed-matched or nonmatched gene sets were analyzed with the Panther gene expression data analysis tool (Thomas et al. 2003, 2006). Enrichment statistics were calculated relative to a custom-loaded background data set consisting of all genes measured in our array profiling. For thermodynamic calculations, seed-matched genes for each miRNA were cross-referenced with hybridization energy calculations derived from the Vienna RNA package (Hofacker 2003) available in the PITA database (Fig. 2B; Supplemental Fig. S2A; Kertesz et al. 2007) or calculated with RNAhybrid (Supplemental Fig. S2B; Kruger and Rehmsmeier 2006). For calculation of target site numbers, all RefSeq (NCBI) transcript sequences associated with genes measured by array profiling that mapped to a unique Ensembl ID were downloaded and searched for 3' UTR seedmatched sequences using MatLab software (Math Works) with code written in-house.

\section{Liver lysates and fractionation}

Prior to harvest, blood was flushed from hepatic tissue by perfusion with $1 \times$ PBS via the portal vein. Lysates were prepared in lysis buffer $(20 \mathrm{mM}$ Tris, $\mathrm{pH} 7.4,100 \mathrm{mM} \mathrm{NaCl}$, and $2.5 \mathrm{mM}$ $\mathrm{MgCl}_{2}$ ) supplemented with EDTA-free protease inhibitor cocktail tablets (Roche). Intact livers ( $\sim 1.0-1.5 \mathrm{~g})$ were homogenized with a glass dounce in $3 \mathrm{~mL}$ ice-cold lysis buffer by twenty strokes with each of the loose and tight plungers. The homogenate was centrifuged at $1000 \mathrm{~g}$ for $10 \mathrm{~min}$ at $4^{\circ} \mathrm{C}$. The resulting S1 supernatant was then centrifuged twice at $16,000 \mathrm{~g}$ for $10 \mathrm{~min}$ at $4^{\circ} \mathrm{C}$. The final S16 supernatant was immediately layered on top of a $20 \%-60 \%$ linear sucrose gradient prepared by gently overlaying decreasing percentages $(\mathrm{w} / \mathrm{v})$ of sucrose solutions prepared in lysis buffer. Equal $\mathrm{OD}_{260}$ units were loaded for each sample. Sucrose gradients were ultracentrifuged at 35,000 rpm, $\sim 218,000 \mathrm{~g}$, for $3 \mathrm{~h}$ at $4^{\circ} \mathrm{C}$ in a Beckman SW41 rotor. After centrifugation, $0.5-\mathrm{mL}$ aliquots were collected by hand by puncturing the bottom of the ultracentrifuge tube. The $A_{260}$ of each fraction was measured with a spectrophotometer. For samples prepared with puromycin, 1 $\mathrm{mM}$ puromycin and $10 \mathrm{mM}$ vanadyl ribonucleoside complex (NEB) were added to the homogenized lysate, the salt concentration was adjusted to $300 \mathrm{mM} \mathrm{NaCl}$, and the lysate was incubated at $37^{\circ} \mathrm{C}$ for $10 \mathrm{~min}$. For samples prepared with EDTA, $0.5 \mathrm{M}$ EDTA, $\mathrm{pH} 8.0$, was added to a final concentration of $30 \mathrm{mM}$. 


\section{miRNA pull-down assay}

miRNA pull-down assays were performed as previously described (Jannot et al. 2011b). Briefly, 2'-O-methyl "capture" oligonucleotides (IDT) modified with a $5^{\prime}$ biotin were titrated into S16 liver lysates and incubated at $25 \mathrm{C}$ for $1 \mathrm{~h}$. Lysates were then incubated with M280 streptavidin-coated magnetic beads (Invitrogen) to precipitate the biotin-conjugated capture strand with its complementary miRNA. Supernatants were removed, and total RNA was purified using Trizol reagent (Invitrogen) and isopropanol precipitation. miRNA was detected using Northern blot with ${ }^{32} \mathrm{P}$ end-labeled complementary DNA probes and 1-ethyl-3-(3-dimethylaminopropyl) carbodiimide (EDC) cross-linking, as previously described (Pall and Hamilton 2008).

\section{mRNA array profiling and analysis}

Total RNA from tissues or cell culture was extracted as per the manufacturer's instructions (miRNeasy kit, Qiagen). mRNA expression profiles were measured for mouse liver and HeLa cells using Mouse Genome 4302.0 arrays (Affymetrix) and U133A arrays (Affymetrix), respectively. mRNA microarrays were run in triplicate for anti-miR- or saline-treated and transgenic mice. For analysis by cumulative distribution frequency, genes containing one or more particular miRNA seed-matched sequences in their RefSeq curated 3' UTRs were compared to those without seeds, and the $P$-value significance of the difference between these curves was determined by a one-sided Kolmogorov-Smirnov test. Webbased software was used to analyze the same data sets using the Sylamer algorithm (van Dongen et al. 2008). Gene expression changes were compared by Student's $t$-test and ranked from highest to lowest fold-change across the $x$-axis. Hypergeometric enrichment scores were computed for all $4^{7}(16,384)$ possible heptamer sequences or for all $4^{8}(65,536)$ possible octamer sequences and were plotted on the $y$-axis.

\section{Oligonucleotide synthesis and purification}

Standard procedure was used to synthesize and purify anti-miRs for miR-21, miR-122, and let-7 used in the current investigation. Briefly, 2'-ribofluoro (2'-F) and 2'-methoxyethyl (MOE) modified oligonucleotides were synthesized on an AKTA Oligopilot 100 (GE Healthcare) synthesizer. The 2'-MOE amidites were acquired from Isis Pharmaceuticals. The F-modified nucleoside phosphoramidites were purchased from ST Pharm and Innovassynth Technologies. The key steps in the solid phase synthesis of F/ MOE anti-miRs 21 and 122 were (1) 3.0 min detritylation step with $15 \%$ DCA/toluene, (2) a $0.15 \mathrm{M}$ solution of the phosphoramidites in anhydrous acetonitrile in each coupling step, and (3) for sulfurization, $0.2 \mathrm{M}$ phenyl acetyl disulfide (PADS) in 1:1 anhydrous pyridine/ $\mathrm{CH} 3 \mathrm{CN}$ was used with 6 -min contact time. After completion of the synthesis, solid support was treated with triethyl amine (TEA):acetonitrile (1:1) at room temperature for $30 \mathrm{~min}$. It was followed by treatment with a mixture of aqueous ammonium hydroxide (33 wt. \%):ethanol (200 proof, Koptec, 3:1) and heated at $55^{\circ} \mathrm{C}$ for $9 \mathrm{~h}$ which resulted in complete removal of all the protecting groups. The anti-miRs were purified by ion exchange chromatography on an AKTA Explorer (GE healthcare) HPLC system on a strong anion exchange column (source $30 \mathrm{Q}$, GE Healthcare). The fractions with high purity (by LCMS) of the desired full-length anti-miR were pooled together and concentrated under high vacuum to a smaller volume. The oligonucleotides were desalted by reverse phase HPLC to furnish desired F/MOE modified anti-miRs in $\sim 40 \%$ isolated yield based on the loading of the solid support. The anti-miRs were lyophilized to a dry powder. The final purity of the anti-miRs was assessed by ion-pair-HPLC-MS analysis with the Agilent 1200 HPLC 6130 MSD system from Agilent Technologies.

\section{RNA analysis}

Following fractionation, total RNA from individual fractions was purified using Trizol (Invitrogen) extraction and miRNeasy-96 kits (Qiagen). RNA was quantified with real-time quantitative PCR, using High Capacity cDNA Reverse Transcription reagents and TaqMan MicroRNA or mRNA assays (Applied Biosystems). RT-qPCR reactions were performed in triplicate in a 384-well format using an Applied Biosystems 7900HT fast real-time PCR instrument. For absolute quantification, standard curves for each amplified miRNA were run in parallel using serial dilutions of synthetic miRNA oligos (IDT) designed from guide-strand sequences listed in the mirBase database (mirbase.org) (GriffithsJones et al. 2006).

\section{Statistical analysis}

Statistical significance was tested as described in the text. ANOVA and nonparametric Kruskal-Wallis tests were calculated using Prism 5.0 (GraphPad Software). One-sided Kolmogorov-Smirnov tests were calculated using the R software package (http://www. $\mathrm{R}$-project.org). Linear regression analysis was calculated using OriginPro 7.0 (OriginLab). For mouse array profiling, genes that showed $\geq 1.10$ fold-change compared to saline treatment with $P<$ 0.05 from three biological replicates per treatment group were considered significant.

\section{3' rapid amplification of cDNA ends (RACE)}

Pdcd4 3' UTR lengths were determined using a RLM-RACE kit (Ambion) and custom nested PCR primers for Pdcd4 (inner Primer: GCACAGCAACTCTTACAGTCTTAGGTGTTAC; outer primer: GAGCTACTGAGCACAGCAACTC). The final PCR product was analyzed with agarose gel electrophoresis.

\section{Western blotting}

Western blot analysis was performed by first pooling together every other fraction and precipitating the protein with the addition of three volumes of prechilled neat ethanol and overnight incubation at $-20^{\circ} \mathrm{C}$. Following centrifugation, the pelleted protein was washed with $70 \%(\mathrm{v} / \mathrm{v})$ cold ethanol and then resuspended in $1 \times$ LDS sample loading buffer (Invitrogen). Due to the high concentration of protein in the top fractions of the gradient (low sucrose density), the first two pooled fractions were resuspended to their original volume prior to precipitation. The remaining fractions were resuspended in 1/10 of their original volume and were thus concentrated $10 \times$. Equal volumes of each pooled fraction were resolved with SDS-PAGE and immunoblotted following a standard protocol. Rabbit polyclonal antibody to Ago2/ eIF2C2 (ab5072) was purchased from Abcam. Rabbit polyclonal antibody to TRBP was developed in-house at Isis Pharmaceuticals. Primary antibodies were detected using IR-dye-labeled secondary 
antibodies, and membranes were scanned using an Odyssey Imaging System (Li-Cor).

\section{SUPPLEMENTAL MATERIAL}

Supplemental material is available for this article.

\section{ACKNOWLEDGMENTS}

We thank S. Orkin and Jochen Hartner (Harvard Medical School) for the generous gift of miR-21 knockout mice, J. Lee and P. Tran for their technical assistance, S. Pitchiaya, E. Marcusson, A. Chang, and D. Hogan for their help and thoughtful insights, and members of Regulus $R \& D$ team and the Walter group for valuable discussions. This work was supported by private funding to Regulus Therapeutics, a Cellular Biotechnology training grant fellowship from the NIH, and a Rackham Graduate School student research grant from the University of Michigan to J.R.A., and NIH grant GM081025 to N.G.W.

Received March 18, 2012; accepted May 23, 2012.

\section{REFERENCES}

Akerfelt M, Morimoto RI, Sistonen L. 2010. Heat shock factors: Integrators of cell stress, development and lifespan. Nat Rev Mol Cell Biol 11: 545-555.

Baek D, Villén J, Shin C, Camargo FD, Gygi SP, Bartel DP. 2008. The impact of microRNAs on protein output. Nature 455: 64-71.

Bartel DP. 2009. MicroRNAs: Target recognition and regulatory functions. Cell 136: 215-233.

Betel D, Koppal A, Agius P, Sander C, Leslie C. 2010. Comprehensive modeling of microRNA targets predicts functional non-conserved and non-canonical sites. Genome Biol 11: R90. doi: 10.1186/gb2010-11-8-r90.

Bhattacharyya SN, Habermacher R, Martine U, Closs EI, Filipowicz W. 2006. Relief of microRNA-mediated translational repression in human cells subjected to stress. Cell 125: 1111-1124.

Bissels U, Wild S, Tomiuk S, Holste A, Hafner M, Tuschl T, Bosio A. 2009. Absolute quantification of microRNAs by using a universal reference. RNA 15: 2375-2384.

Burchard J, Zhang C, Liu AM, Poon RT, Lee NP, Wong KF, Sham PC, Lam BY, Ferguson MD, Tokiwa G, et al. 2010. microRNA-122 as a regulator of mitochondrial metabolic gene network in hepatocellular carcinoma. Mol Syst Biol 6: 402. doi: 10.1038/msb.2010.58.

Burns DM, D'Ambrogio A, Nottrott S, Richter JD. 2011. CPEB and two poly(A) polymerases control miR-122 stability and p53 mRNA translation. Nature 473: 105-108.

Cesana M, Cacchiarelli D, Legnini I, Santini T, Sthandier O, Chinappi M, Tramontano A, Bozzoni I. 2011. A long noncoding RNA controls muscle differentiation by functioning as a competing endogenous RNA. Cell 147: 358-369.

Cheloufi S, Dos Santos CO, Chong MMW, Hannon GJ. 2010. A dicerindependent miRNA biogenesis pathway that requires Ago catalysis. Nature 465: 584-589.

Davis S, Propp S, Freier SM, Jones LE, Serra MJ, Kinberger G, Bhat B, Swayze EE, Bennett CF, Esau C. 2009. Potent inhibition of microRNA in vivo without degradation. Nucleic Acids Res 37: $70-77$.

Edgar R, Domrachev M, Lash AE. 2002. Gene Expression Omnibus: NCBI gene expression and hybridization array data repository. Nucleic Acids Res 30: 207-210.

Elmén J, Lindow M, Silahtaroglu A, Bak M, Christensen M, LindThomsen A, Hedtjärn M, Hansen JB, Hansen HF, Straarup EM, et al. 2008. Antagonism of microRNA-122 in mice by systemically administered LNA-antimiR leads to up-regulation of a large set of predicted target mRNAs in the liver. Nucleic Acids Res 36: 11531162.

Fabian MR, Svitkin YV, Sonenberg N. 2011. An efficient system for Let-7 microRNA and GW182 protein-mediated deadenylation in vitro. Methods Mol Biol 725: 207-217.

Filipowicz W, Grosshans H. 2011. The liver-specific microRNA miR122: Biology and therapeutic potential. Prog Drug Res 67: 221-238.

Frankel LB, Christoffersen NR, Jacobsen A, Lindow M, Krogh A, Lund AH. 2008. Programmed cell death 4 (PDCD4) is an important functional target of the microRNA miR-21 in breast cancer cells. J Biol Chem 283: 1026-1033.

Friedman RC, Farh KK-H, Burge CB, Bartel DP. 2009. Most mammalian mRNAs are conserved targets of microRNAs. Genome Res 19: 92-105.

Garcia DM, Baek D, Shin C, Bell GW, Grimson A, Bartel DP. 2011. Weak seed-pairing stability and high target-site abundance decrease the proficiency of $l s y-6$ and other microRNAs. Nat Struct Mol Biol 18: 1139-1146.

Griffiths-Jones S, Grocock RJ, van Dongen S, Bateman A, Enright AJ. 2006. miRBase: MicroRNA sequences, targets and gene nomenclature. Nucleic Acids Res 34: D140-D144.

Grimson A, Farh K, Johnston W, Garrett-Engele P, Lim L, Bartel DP. 2007. MicroRNA targeting specificity in mammals: Determinants beyond seed pairing. Mol Cell 27: 91-105.

Guo H, Ingolia NT, Weissman JS, Bartel DP. 2010. Mammalian microRNAs predominantly act to decrease target mRNA levels. Nature 466: 835-840.

Harper LV, Hilton AC, Jones AF. 2003. RT-PCR for the pseudogenefree amplification of the glyceraldehyde-3-phosphate dehydrogenase gene (gapd). Mol Cell Probes 17: 261-265.

Hatley ME, Patrick DM, Garcia MR, Richardson JA, Bassel-Duby R, van Rooij E, Olson EN. 2010. Modulation of K-ras-dependent lung tumorigenesis by microRNA-21. Cancer Cell 18: 282-293.

Hendrickson DG, Hogan DJ, McCullough HL, Myers JW, Herschlag D, Ferrell JE, Brown PO. 2009. Concordant regulation of translation and mRNA abundance for hundreds of targets of a human microRNA. PLoS Biol 7: e1000238. doi: 10.1371/journal.pbio.1000238.

Hofacker IL. 2003. Vienna RNA secondary structure server. Nucleic Acids Res 31: 3429-3431.

Jackson R, Standart N. 2007. How do microRNAs regulate gene expression? Sci STKE 2007: re1. doi: 10.1126/stke.3672007re1.

Jannot G, Bajan $S$, Giguère NJ, Bouasker $S$, Banville $I H$, Piquet $S$, Hutvagner G, Simard MJ. 2011a. The ribosomal protein RACK1 is required for microRNA function in both $C$. elegans and humans. EMBO Rep 12: 581-586.

Jannot G, Vasquez-Rifo A, Simard MJ. 2011b. Argonaute pull-down and RISC analysis using 2'-O-methylated oligonucleotides affinity matrices. Methods Mol Biol 725: 233-249.

Jazbutyte V, Thum T. 2010. MicroRNA-21: From cancer to cardiovascular disease. Curr Drug Targets 11: 926-935.

Kertesz M, Iovino N, Unnerstall U, Gaul U, Segal E. 2007. The role of site accessibility in microRNA target recognition. Nat Genet 39: $1278-1284$.

Kim J, Krichevsky A, Grad Y, Hayes GD, Kosik KS, Church GM, Ruvkun G. 2004. Identification of many microRNAs that copurify with polyribosomes in mammalian neurons. Proc Natl Acad Sci 101: 360-365.

Krek A, Grün D, Poy MN, Wolf R, Rosenberg L, Epstein EJ, MacMenamin P, da Piedade I, Gunsalus KC, Stoffel M, et al. 2005. Combinatorial microRNA target predictions. Nat Genet 37: 495-500.

Kruger J, Rehmsmeier M. 2006. RNAhybrid: microRNA target prediction easy, fast and flexible. Nucleic Acids Res 34: W451-W454.

Krützfeldt J, Rajewsky N, Braich R, Rajeev KG, Tuschl T, Manoharan M, Stoffel M. 2005. Silencing of microRNAs in vivo with 'antagomirs'. Nature 438: 685-689.

Lambert NJ, Gu SG, Zahler AM. 2011. The conformation of microRNA seed regions in native microRNPs is prearranged for presentation to mRNA targets. Nucleic Acids Res 39: 4827-4835. 
Lewis B, Shih I, Jones-Rhoades M, Bartel DP, Burge C. 2003 Prediction of mammalian microRNA targets. Cell 115: 787-798.

Liu C, Yu J, Yu S, Lavker RM, Cai L, Liu W, Yang K, He X, Chen S. 2010. MicroRNA-21 acts as an oncomir through multiple targets in human hepatocellular carcinoma. J Hepatol 53: 98-107.

Lu M, Zhang Q, Deng M, Miao J, Guo Y, Gao W, Cui Q. 2008. An analysis of human microRNA and disease associations. PLoS ONE 3: e3420. doi: 10.1371/journal.pone.0003420.

Lu TX, Hartner J, Lim E-J, Fabry V, Mingler MK, Cole ET, Orkin SH, Aronow BJ, Rothenberg ME. 2011. MicroRNA-21 limits in vivo immune response-mediated activation of the IL- $12 / \mathrm{IFN}-\gamma$ pathway, Th1 polarization, and the severity of delayed-type hypersensitivity. J Immunol 187: 3362-3373.

Lui WO, Pourmand N, Patterson BK, Fire A. 2007. Patterns of known and novel small RNAs in human cervical cancer. Cancer Res 67: 6031-6043.

MacRae IJ, Ma E, Zhou M, Robinson CV, Doudna JA. 2008. In vitro reconstitution of the human RISC-loading complex. Proc Natl Acad Sci 105: 512-517.

Majlessi M, Nelson NC, Becker MM. 1998. Advantages of 2'$O$-methyl oligoribonucleotide probes for detecting RNA targets. Nucleic Acids Res 26: 2224-2229.

Mallanna SK, Rizzino A. 2010. Emerging roles of microRNAs in the control of embryonic stem cells and the generation of induced pluripotent stem cells. Dev Biol 344: 16-25.

Manche L, Green SR, Schmedt C, Mathews MB. 1992. Interactions between double-stranded RNA regulators and the protein kinase DAI. Mol Cell Biol 12: 5238-5348.

Maroney PA, Yu Y, Fisher J, Nilsen TW. 2006. Evidence that microRNAs are associated with translating messenger RNAs in human cells. Nat Struct Mol Biol 13: 1102-1107.

Mayr C, Hemann MT, Bartel DP. 2007. Disrupting the pairing between let-7 and Hmga2 enhances oncogenic transformation. Science 315: 1576-1579.

Medina PP, Nolde M, Slack FJ. 2010. OncomiR addiction in an in vivo model of microRNA-21-induced pre-B-cell lymphoma. Nature 467: 86-90.

Mehan S, Meena H, Sharma D, Sankhla R. 2011. JNK: A stressactivated protein kinase therapeutic strategies and involvement in Alzheimer's and various neurodegenerative abnormalities. J Mol Neurosci 43: $376-390$.

Meng F, Henson R, Wehbe-Janek H, Ghoshal K, Jacob ST, Patel T. 2007. MicroRNA-21 regulates expression of the PTEN tumor suppressor gene in human hepatocellular cancer. Gastroenterology 133: 647-658.

Munz C, Psichari E, Mandilis D, Lavigne A-C, Spiliotaki M, Oehler T, Davidson I, Tora L, Angel P, Pintzas A. 2003. TAF7 $\left(\mathrm{TAF}_{\mathrm{II}} 55\right)$ plays a role in the transcription activation by c-Jun. J Biol Chem 278: $21510-21516$.

Nelson PT, Hatzigeorgiou AG, Mourelatos Z. 2004. miRNP:mRNA association in polyribosomes in a human neuronal cell line. RNA 10: $387-394$.

Nottrott S, Simard MJ, Richter JD. 2006. Human let-7a miRNA blocks protein production on actively translating polyribosomes. Nat Struct Mol Biol 13: 1108-1114.

Pall GS, Hamilton AJ. 2008. Improved northern blot method for enhanced detection of small RNA. Nat Protoc 3: 1077-1084.

Patrick DM, Montgomery RL, Qi X, Obad S, Kauppinen S, Hill JA, van Rooij E, Olson EN. 2010. Stress-dependent cardiac remodeling occurs in the absence of microRNA-21 in mice. J Clin Invest 120: 3912-3916.

Pham J, Pellino J, Lee Y, Carthew R, Sontheimer EJ. 2004. A Dicer2-dependent $80 \mathrm{~S}$ complex cleaves targeted mRNAs during RNAi in Drosophila. Cell 117: 83-94.

Pillai RS, Bhattacharyya SN, Artus CG, Zoller T, Cougot N, Basyuk E, Bertrand E, Filipowicz W. 2005. Inhibition of translational initiation by let-7 microRNA in human cells. Science 309: 1573-1576.

Pink RC, Wicks K, Caley DP, Punch EK, Jacobs L, Carter DRF. 2011. Pseudogenes: Pseudo-functional or key regulators in health and disease? RNA 17: 792-798.
Poliseno L, Salmena L, Zhang J, Carver B, Haveman WJ, Pandolfi PP. 2010. A coding-independent function of gene and pseudogene mRNAs regulates tumour biology. Nature 465: 10331038.

Reynolds A, Anderson EM, Vermeulen A, Fedorov Y, Robinson K, Leake D, Karpilow J, Marshall WS, Khvorova A. 2006. Induction of the interferon response by siRNA is cell type- and duplex length-dependent. RNA 12: 988-993.

Roush S, Slack FJ. 2008. The let-7 family of microRNAs. Trends Cell Biol 18: 505-516.

Sampson J, Mathews MB, Osborn M, Borghetti AF. 1972. Hemoglobin messenger ribonucleic acid translation in cell-free systems from rat and mouse liver and Landschutz ascites cells. Biochemistry 11: 3636-3640.

Schmittgen TD, Livak KJ. 2008. Analyzing real-time PCR data by the comparative $C_{\mathrm{T}}$ method. Nat Protoc 3: 1101-1108.

Selbach M, Schwanhausser B, Thierfelder N, Fang Z, Khanin R, Rajewsky N. 2008. Widespread changes in protein synthesis induced by microRNAs. Nature 455: 58-63.

Sumazin P, Yang X, Chiu H-S, Chung W-J, Iyer A, Llobet-Navas D, Rajbhandari P, Bansal M, Guarnieri P, Silva J, et al. 2011. An extensive microRNA-mediated network of RNA-RNA interactions regulates established oncogenic pathways in glioblastoma. Cell 147: 370-381.

Tay Y, Kats L, Salmena L, Weiss D, Tan SM, Ala U, Karreth F, Poliseno L, Provero P, Di Cunto F, et al. 2011. Coding-independent regulation of the tumor suppressor PTEN by competing endogenous mRNAs. Cell 147: 344-357.

Thomas PD, Kejariwal A, Campbell MJ, Mi H, Diemer K, Guo N, Ladunga I, Ulitsky-Lazareva B, Muruganujan A, Rabkin S, et al. 2003. PANTHER: A browsable database of gene products organized by biological function, using curated protein family and subfamily classification. Nucleic Acids Res 31: 334341.

Thomas PD, Kejariwal A, Guo N, Mi H, Campbell MJ, Muruganujan A, Lazareva-Ulitsky B. 2006. Applications for protein sequencefunction evolution data: mRNA/protein expression analysis and coding SNP scoring tools. Nucleic Acids Res (Suppl. 2) 34: W645W650.

Thum T, Gross C, Fiedler J, Fischer T, Kissler S, Bussen M, Galuppo P, Just S, Rottbauer W, Frantz S, et al. 2008. MicroRNA-21 contributes to myocardial disease by stimulating MAP kinase signalling in fibroblasts. Nature 456: 980-984.

Turner NA. 2011. Therapeutic regulation of cardiac fibroblast function: Targeting stress-activated protein kinase pathways. Future Cardiol 7: 673-691.

van Dongen S, Abreu-Goodger C, Enright AJ. 2008. Detecting microRNA binding and siRNA off-target effects from expression data. Nat Methods 5: 1023-1025.

Vecchione A, Croce CM. 2010. Apoptomirs: Small molecules have gained the license to kill. Endocr Relat Cancer 17: F37-F50.

Volinia S, Calin GA, Liu C-G, Ambs S, Cimmino A, Petrocca F, Visone R, Iorio M, Roldo C, Ferracin M, et al. 2006. A microRNA expression signature of human solid tumors defines cancer gene targets. Proc Natl Acad Sci 103: 2257-2261.

Wang B, Love TM, Call ME, Doench JG, Novina CD. 2006. Recapitulation of short RNA-directed translational gene silencing in vitro. Mol Cell 22: 553-560.

Xiao F, Zuo Z, Cai G, Kang S, Gao X, Li T. 2009. miRecords: An integrated resource for microRNA-target interactions. Nucleic Acids Res 37: D105-D110.

Yao Q, Xu H, Zhang QQ, Zhou H, Qu LH. 2009. MicroRNA-21 promotes cell proliferation and down-regulates the expression of programmed cell death 4 (PDCD4) in HeLa cervical carcinoma cells. Biochem Biophys Res Commun 388: 539-542.

Yuan CX, Gurley WB. 2000. Potential targets for HSF1 within the preinitiation complex. Cell Stress Chaperones 5: 229-242.

Zhao Y, Srivastava D. 2007. A developmental view of microRNA function. Trends Biochem Sci 32: 189-197. 

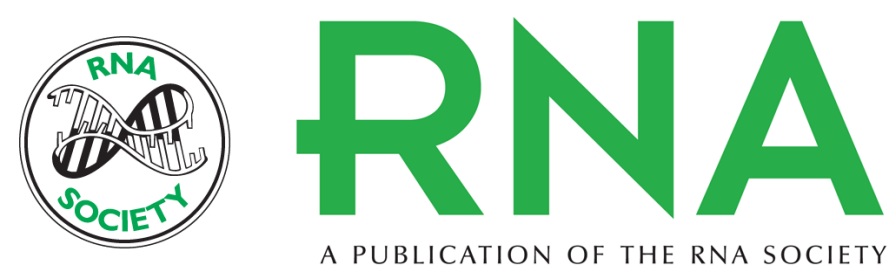

\section{Disease-linked microRNA-21 exhibits drastically reduced mRNA binding and silencing activity in healthy mouse liver}

John R. Androsavich, B. Nelson Chau, Balkrishen Bhat, et al.

RNA 2012 18: 1510-1526 originally published online June 27, 2012

Access the most recent version at doi:10.1261/rna.033308.112

Supplemental http://rnajournal.cshlp.org/content/suppl/2012/06/06/rna.033308.112.DC1
Material

References This article cites 77 articles, 17 of which can be accessed free at: http://rnajournal.cshlp.org/content/18/8/1510.full.html\#ref-list-1

License

Email Alerting Receive free email alerts when new articles cite this article - sign up in the box at the Service top right corner of the article or click here.

To subscribe to $R N A$ go to:

http://rnajournal.cshlp.org/subscriptions 